Management international

International Management

Gestiòn Internacional

\title{
Quel est le rôle du substrat technique dans l'appropriation d'une innovation managériale ? Une analyse longitudinale d'une méthode innovante
}

\section{What Role Does the Technical Substrate Play in the Appropriation of Management Innovation? A Longitudinal Analysis of an Innovative Method \\ ¿Qué papel desempeña el substrato técnico en la apropiación de una innovación de gestión? Un análisis longitudinal de un método innovador}

\section{Emilie CANET et Sébastien TRAN}

Volume 21, numéro 4, été 2017

URI : https://id.erudit.org/iderudit/1053576ar

DOI : https://doi.org/10.7202/1053576ar

Aller au sommaire du numéro

Éditeur(s)

HEC Montréal

Université Paris Dauphine

ISSN

1206-1697 (imprimé)

1918-9222 (numérique)

Découvrir la revue

Citer cet article

CANET, E. \& TRAN, S. (2017). Quel est le rôle du substrat technique dans l'appropriation d'une innovation managériale ? Une analyse longitudinale d'une méthode innovante. Management international / International Management / Gestiòn Internacional, 21(4), 28-47.

https://doi.org/10.7202/1053576ar

\section{Résumé de l'article}

Cette recherche propose une réflexion sur l'appropriation d'une innovation managériale par les acteurs de l'organisation qui la conçoivent. Parce que le substrat technique est l'élément concret qui matérialise l'innovation, nous analysons son rôle dans ce processus. Notre recherche est basée sur l'étude longitudinale du cas d'une innovation managériale au sein d'un grand groupe industriel. Cette recherche permet de montrer que la construction de l'innovation managériale repose sur une interaction forte entre substrat technique et philosophie gestionnaire. L'appropriation est également assurée par la légitimité émergente de nouveaux acteurs promue par les nouvelles relations favorisées par l'innovation.
Tous droits réservés (C) Management international / International Management / Gestión Internacional, 2017
Ce document est protégé par la loi sur le droit d'auteur. L’utilisation des services d’Érudit (y compris la reproduction) est assujettie à sa politique d'utilisation que vous pouvez consulter en ligne. 


\title{
Quel est le rôle du substrat technique dans l'appropriation d'une innovation managériale? Une analyse longitudinale d'une méthode innovante ${ }^{*}$
}

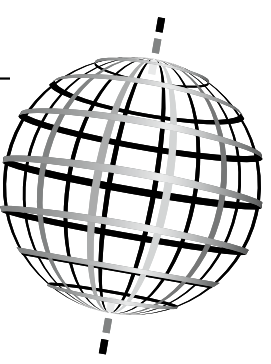

\author{
What Role Does the Technical Substrate Play in \\ the Appropriation of Management Innovation? \\ A Longitudinal Analysis of an Innovative Method ${ }^{*}$
}

\section{¿Qué papel desempeña el substrato técnico en la apropiación de una innovación de gestión? Un análisis longitudinal de un método innovador*}

\author{
EMILIE CANET \\ Université Paris-Dauphine, PSL Research University, \\ CNRS, UMR 7088, DRM (M-Lab), 75016, Paris
}

\author{
SÉBASTIEN TRAN \\ Léonard de Vinci Pôle Universitaire, \\ Research Center (DVRC), 92916 Paris La Défense, France
}

\section{RÉSUMÉ}

Cette recherche propose une réflexion sur l'appropriation d'une innovation managériale par les acteurs de l'organisation qui la conçoivent. Parce que le substrat technique est l'élément concret qui matérialise l'innovation, nous analysons son rôle dans ce processus. Notre recherche est basée sur l'étude longitudinale du cas d'une innovation managériale au sein d'un grand groupe industriel. Cette recherche permet de montrer que la construction de l'innovation managériale repose sur une interaction forte entre substrat technique et philosophie gestionnaire. L'appropriation est également assurée par la légitimité émergente de nouveaux acteurs promue par les nouvelles relations favorisées par l'innovation.

Mots clés : innovation managériale, appropriation, substrat technique, philosophie gestionnaire.

\section{ABSTRACT}

This research is focused on the appropriation process in management innovation. As the technical substrate is the physical medium in which the innovation materializes, we analyze its role in this process. Our research is based on a longitudinal analysis of management innovation in a large industrial group. We demonstrate that the creation of managerial innovation rests on a strong interaction between the technical substrate and a managerial philosophy. The legitimate rise of new actors, integrated in a new relationship system thanks to managerial innovation, reinforces the appropriation process.

Keywords: management innovation, appropriation, technical substrate, managerial philosophy.

\section{RESUMEN}

Esta investigación propone una reflexión sobre el proceso de apropiación de una innovación de gestión por sus actores creadores. Dado que el substrato técnico materializa la innovación, analizamos su papel en dicho proceso. Nuestra investigación se basa en el estudio longitudinal de una innovación de gestión en un gran grupo industrial. Demostramos que la innovación en gestión se fundamenta en una sólida interacción entre el substrato técnico y la filosofía de gestión. Asimismo, la apropiación de la innovación se basa en la integración legítima de nuevos actores cuya adhesión a las nuevas relaciones empresariales contribuye a incentivar la innovación.

Palabras clave: innovación de gestión, apropiación, substrato técnico, filosofía de gestión.

\begin{abstract}
T 'innovation managériale est au cœur de nombreuses réflexions U(Kimberly, 1981; Damanpour et Evan, 1984; Wolfe, 1994; Abrahamson, 1996; Birkinshaw et al., 2008) mais reste encore relativement peu explorée, conceptuellement comme empiriquement, au regard des autres types d'innovations (Damanpour et Aravind, 2012). Elle apparaît pourtant comme le principal facteur d'explication de la performance et donc la solution pour créer des avantages concurrentiels (Hamel, 2006, 2009; Mol et Birkinshaw, 2009; Le Roy et al., 2013). Les entreprises sont de plus en plus nombreuses à introduire ou à adopter des innova-
\end{abstract}

tions managériales, en complément des innovations produits mais leur dimension tacite n'est pas sans poser des problèmes (Birkinshaw et al., 2008; Volderba et al., 2014). La réussite du processus d'innovation managériale implique nécessairement une appropriation de celle-ci par les acteurs impliqués dans l'organisation. En effet, l'innovation mise en œuvre s'intègre, au fur et mesure de son expérimentation, dans les routines de l'organisation. Cette intégration se produit dans un contexte de création du sens à donner à l'innovation au fil de sa conception (Alter, 2000; Grimand, 2012) afin de faciliter son appropriation

* Nous tenons à remercier Ibrahima Fall et Philippe Rauffet pour l'accès à leurs matériaux de recherche et aux nombreux échanges que nous avons pu avoir avec eux. 1. Rapport de l'OECD : «Innovation microdata project based on CIS-2006, June 2009 and national data sources » 
par les différents acteurs. La définition de l'innovation est le résultat des forces en présence au cours du processus, et notamment du concepteur et des utilisateurs, notamment parce que le concepteur favorise un contexte de modification de l'innovation par ses utilisateurs pour favoriser son appropriation (Ansari et al., 2014; Peeters et al., 2014). Cette construction de l'innovation managériale passe par la définition de ses trois composantes : substrat technique, philosophie gestionnaire et vision simplifiée des relations organisationnelles (Hatchuel et Weil, 1992; David, 1996). La philosophie gestionnaire, cœur de l'innovation managériale, est particulièrement difficile à saisir pour les acteurs de l'organisation et de nombreuses difficultés d'appropriation résident dans son appréhension (Mamman, 2009). Parce que le substrat technique est le support qui matérialise l'innovation et qui reflète, en partie, cette philosophie, il convient de réfléchir à son rôle dans l'appropriation de l'innovation par les acteurs de l'organisation, face aux difficultés rencontrées. Cela permet de réintroduire la dimension matérielle au cœur de l'analyse de l'activité organisationnelle, comme le suggèrent les travaux récents sur la sociomatérialité (Orlikowski et Scott, 2008; Orlikowski, 2010; Leonardi, 2013).

Cette recherche s'appuie sur l'analyse longitudinale d'une innovation managériale, depuis l'émergence de l'idée jusqu'à sa mise en œuvre effective au sein d'une grande organisation industrielle et internationale entre 2005 et 2010. Cette innovation repose sur un substrat technique composé d'un Système d'Information (SI) qui soutient sa mise en œuvre. Cette analyse processuelle permet d'éclairer le processus d'innovation managériale de manière complète en nous concentrant sur les enjeux de l'appropriation et en mettant en lumière le rôle du substrat technique. Nous présentons dans une première partie les enjeux liés au processus d'innovation managériale et plus particulièrement les difficultés de son appropriation. Nous voyons également dans quelle mesure une attention particulière doit être portée sur la dimension matérielle de l'innovation pour assurer son appropriation. Puis nous présentons notre méthodologie qualitative, en mettant en évidence les résultats, avant de les discuter dans une quatrième partie.

\section{Les innovations managériales : un mécanisme d'appropriation complexe}

\section{DÉFINITION ET PROPRIÉTÉS DES INNOVATIONS MANAGÉRIALES}

L'innovation managériale est une forme d'innovation qui reste encore relativement peu explorée, conceptuellement comme empiriquement, au regard des autres types d'innovations (Damanpour et Aravind, 2012). Plusieurs termes sont utilisés pour parler de ces innovations : organisationnelles (Edquist et al., 2001; Sanidas, 2005; Dubouloz et Bocquet, 2013), administratives (Damanpour et Evan, 1984; Gosselin, 1997; Ravichandran, 2000) ou encore managériales (Kimberly, 1981; Hamel, 2006; Birkinshaw et al., 2008). Toutefois, leur définition se recoupe de manière significative (Damanpour et Aravind, 2012). Ces innovations sont largement conceptualisées en les distinguant des autres types d'innovations que sont les innovations de produit, de service et surtout technologiques (Damanpour et Aravind, 2012). Elles se réfèrent à la sphère sociale de l'organisation en opposition à la sphère technologique, en imposant des modifications dans les processus de management et des relations entre acteurs de l'organisation pour réaliser les activités (Le Roy et al., 2013). Alors que certains considèrent l'innovation managériale comme un produit (Kimberly, 1981; Van de Ven, 1986), d'autres travaux la caractérisent par une dimension processuelle, à savoir un encastrement progressif dans les pratiques de l'organisation (Birkinshaw et al., 2008). L'innovation managériale est alors définie comme «l'invention et la mise en œuvre d'une pratique, d'un processus, d'une structure ou d'une technique managériale, qui est nouvelle par rapport à l'état de l'art et qui vise à prolonger les objectifs de l'organisation» (Birkinshaw et al., 2008). Les exemples d'innovations managériales que sont la forme multi-divisionnelle, le management de la qualité totale, le Balanced Scorecard ou encore l'Open-Innovation se caractérisent ainsi par un niveau opérationnel. L'innovation managériale modifie de façon concrète le travail et les pratiques des managers (Hamel, 2006; Le Roy et al., 2013) et elle s'insère dans un objectif d'amélioration de la performance de l'organisation (Hamel, 2006; Mol et Birkinshaw, 2009; Damanpour et Aravind, 2012).

Le processus d'innovation managériale est récursif et composé de 4 phases (Birkinshaw et al., 2008). Il s'inscrit dans un cycle répété de variation, sélection et rétention de nouvelles pratiques (Zbaracki, 1998), régulé par la capacité d’absorption interne des organisations (Peeters et al., 2014). Les acteurs, confrontés à un problème, ressentent une motivation à expérimenter une innovation managériale. A travers des variations planifiées ou non intentionnelles, l'innovation émerge et les acteurs la font évoluer par un processus d'essai-erreur. L'innovation managériale qui va émerger, après plusieurs itérations, sera un compromis jugé acceptable par les acteurs de l'organisation qui auront négocié son sens et son usage pour qu'elle se plie à leurs intérêts (Alter, 2000; Segrestin, 2004). Lorsque le processus d'innovation managériale réussit, il conduit donc à l'émergence d'une innovation institutionnalisée, théorisée et étiquetée (Birkinshaw et al., 2008). Celle-ci a non seulement été intégrée dans les pratiques et les routines de l'organisation, mais elle a aussi été l'objet d'un travail d'explicitation et de formalisation. $\mathrm{Au}$-delà de son expérimentation, il s'agit de donc construire et de définir l'innovation, ses caractéristiques, son utilisation.

L'introduction d'une innovation managériale au sein d'une organisation est comparable à toute situation de changement (David, 1998; Segrestin, 2004; Damanpour et Aravind, 2012), qui touche aux routines de l'organisation (Birkinshaw et al., 2008; Peeters et al., 2014). A ce titre, cette introduction va provoquer des transformations de l'innovation par l'organisation et réciproquement (David, 1998). En effet, l'introduction d'un élément nouveau dans l'organisation crée à la fois de l'ambiguïté pour les acteurs, qui ont une compréhension incomplète de la valeur attendue de l'innovation, et de l'incertitude par la crainte de conséquences négatives pour les acteurs ou l'organisation (Birkinshaw et al., 2008). Ainsi, l'innovation apparaît souvent comme «déraisonnable» parce qu'elle est en rupture par rapport aux pratiques établies, et elle va donc se construire sur le vide et l'ambiguïté (Alter, 2000). La réussite du processus d'innovation managériale implique donc que celle-ci soit reconnue et acceptée par les acteurs de l'organisation, en d'autres termes «toute prescription managériale appelle une appropriation par 
le bas» (Segrestin, 2004, p. 23). L'innovation mise en œuvre doit s'intégrer, au fur et mesure de son expérimentation, dans les routines $^{2}$ de l'organisation. Cette intégration se produit dans un contexte de création du sens à donner à l'innovation au fil de sa conception. Ainsi, les processus d'innovation managériale et d'appropriation sont, en partie, confondus. En effet, le processus d'innovation managériale n'est complet que lorsque la pratique managériale inventée est intégrée dans les routines de l'organisation, et donc que les différents acteurs de l'organisation sont parvenus à définir son sens et son usage. Comme le soulignent Peeters et al. (2014), l'innovation managériale est permise par la capacité d'absorption interne des organisations qui contribue à faciliter la variation, favoriser l'émergence de nouvelles idées, les sélectionner et enfin permettre leur intégration dans les routines de l'organisation.

\section{LES ENJEUX DU PROCESSUS D'APPROPRIATION DES INNOVATIONS MANAGÉRIALES}

Les travaux récents sur l'innovation managériale présentent l'appropriation comme un processus multidimensionnel (Damanpour et Schneider, 2006; Peeters et al., 2014), qui est influencé par de nombreux facteurs internes comme externes (facteurs organisationnels, culture, rôle des acteurs, acteurs externes, influence de l'environnement, etc.). La notion d'appropriation est une notion qui recouvre deux acceptions (Mallet, 2006). Elle signifie à la fois «rendre propre à un usage précis» mais recouvre aussi l'idée de «s'attribuer» quelque chose et d'en faire sa "propriété». Cette notion d'appropriation est couramment utilisée pour comprendre l'appropriation des outils et dispositifs de gestion au sein des organisations (de Vaujany et Grimand, 2005; de Vaujany, 2006; Grimand, 2012). Elle peut être mobilisée pour les innovations managériales (Hatchuel, 2000; Segrestin, 2004), dans la mesure où ces innovations modifient de manière concrète la manière de travailler des acteurs de l'organisation.

Le processus d'appropriation passe par trois phénomènes (Proulx, 2001; Grimand, 2012). Tout d'abord, l'appropriation implique une maîtrise croissante de l'innovation par les acteurs de l'organisation, qui doivent comprendre et maîtriser les savoirs et compétences nécessaires à l'utilisation de l'innovation (Proulx, 2001), ce qui implique d'adopter de nouveaux principes d'action (Segrestin, 2004). Ainsi, l'appropriation est considérée comme complète dès lors qu'il y a une intégration réelle dans les routines des acteurs. L'appropriation aboutit à la formation de routines stabilisées incluant l'innovation. Enfin, les acteurs créent du sens et font évoluer l'innovation pour assurer cette appropriation. Les apprentissages des acteurs conduisent à certaines interprétations, précisions ou encore reconstructions. Cela impose donc de considérer l'appropriation comme un processus interprétatif (Ségrestin, 2004). Les acteurs négocient entre eux et construisent le sens et la représentation partagée de l'innovation. En effet, la rencontre de l'innovation avec les acteurs de terrain provoque des tensions qui vont modifier le projet initial et implique nécessairement un acte de création (Segrestin, 2004). La réussite de l'appropriation dépend donc d'acteurs qui parviennent à détecter les opportunités de l'innovation compatibles avec leurs intérêts, voire de faire plier l'innovation à leurs intérêts (Segrestin, 2004).

La compréhension de l'appropriation, et cette nécessaire création de sens entre acteurs, empêche donc de penser conception et usage séparément (Lorino, 2002; de Vaujany et Grimand, 2005). En effet, la mise en œuvre de l'innovation intègre nécessairement une activité de conception dans laquelle l'innovation prend réellement son sens au regard des spécificités et contextes de l'organisation dans laquelle elle se déploie. L'innovation n'existe donc que parce qu'elle est mise en œuvre. De Sanctis et Poole (1994) considère également que l'appropriation est l'acte d'amener les règles et les ressources dans l'action. De plus, les connaissances autour de l'innovation assurant l'appropriation sont nécessairement construites par les acteurs qui l'utilisent et participent à l'existence de l'innovation dans l'organisation (Lorino, 2002). L'ensemble des acteurs impliqués dans le processus d'innovation managériale participe donc à la conception permanente de l'innovation ${ }^{3}$.

On peut s'interroger sur la spécificité de l'appropriation d'une innovation managériale. La nature tacite de l'innovation managériale génère une interprétation plus subjective par ses utilisateurs (Peeters et al., 2014). Ce flou relatif peut cependant favoriser l'appropriation parce qu'il crée justement un espace de création de sens pour les acteurs de l'organisation (Ansari et al., 2014). Mais cette construction de sens sera d'autant plus complexe que l'objet est nouveau, en rupture avec les pratiques et routines existantes. Dans le cas des innovations managériales les plus radicales, il s'agit même de trouver de nouveaux termes pour pouvoir décrire ces nouveautés. On assiste donc à une véritable construction simultanée du sens au fur et à mesure de l'usage qui s'inscrit dans une logique d'expérimentation. Par exemple, les pratiques mises en œuvre par Ohno au sein de Toyota à la fin des années 1970 ont abouti à la définition d'outils et de principes formalisés et l'invention de l'expression "lean management ${ }^{4}$. A ce titre, l'appropriation d'une innovation managériale peut aller jusqu'à remettre en cause la manière même de penser son management. Comme le souligne Alter (2000), l'appropriation de l'innovation managériale est difficile parce qu'elle n'a justement pas de sens bien déterminé pour les acteurs au départ. Ainsi, au moins momentanément, cette appropriation se fait contre l'ordre organisationnel et les pratiques établies. Cette innovation va ensuite modifier l'organisation et les rôles habituellement affectés aux acteurs (Alter, 2000). C'est donc dans un nouveau contexte organisationnel que les acteurs doivent parvenir à construire son sens.

Concepteurs et managers jouent un rôle important dans l'appropriation des innovations managériales en favorisant un espace de liberté dans l'utilisation de l'innovation qui permet

2. Nous définissons le concept de routine comme des conduites ou des pratiques effectives directement observables (fixation des prix, décisions de gestion, décisions d'investissement, etc.) qui peuvent expliquer au moins partiellement les performances des différentes firmes et la transformation de ces performances, notamment lorsqu'elles sont modifiées d'une manière ou d'une autre par l'innovation (Mangolte, 1998).

3. Par souci de simplification, nous utilisons par la suite le terme de «concepteurs" pour désigner les initiateurs de l'innovation managériale, qui portent la vision et l'intention initiales de l'innovation; même si cette innovation est en réalité co-conçue par l'ensemble des acteurs impliqués dans sa mise en œuvre.

4. Ce terme marque la rupture et l'opposition avec les pratiques alors en œuvre dans l'industrie automobile et promeut un mode de fonctionnement évitant le gaspillage et fondé sur la montée en responsabilité des opérateurs. 
la création de sens pour les acteurs de l'organisation (Alter, 2000; Ansari et al., 2014; Peeters et al., 2014). L'organisation doit trouver un équilibre entre la standardisation des pratiques et l'adaptation locale possible (Ansari et al., 2014 ). En d'autres termes, le concepteur de l'innovation doit encourager la modification de l'innovation lorsqu'elle est potentiellement bénéfique pour l'organisation, et parce qu'elle favorise la création de sens et donc l'appropriation, tout en s'assurant que cette modification ne conduise pas à un éloignement trop important par rapport à la philosophie gestionnaire de départ. On retrouve cette idée dans les travaux de Peeters et al. (2006) qui démontrent que l'appropriation passe par la création par le management d'un contexte qui encourage l'adaptation, dans lequel la capacité clé est celle du partage de la connaissance entre acteurs. Deux logiques antagoniques s'opposent donc (Alter, 2000) : celle de l'innovation contre celle de l'organisation, et c'est leur rencontre qui permet de donner du sens à l'invention initiale. L'innovation tire parti des incertitudes et elle va repréciser les rôles de chacun au fur et à mesure qu'elle se développe.

\section{SUBSTRAT TECHNIQUE DE L'INNOVATION MANAGÉRIALE ET APPROPRIATION}

La construction du sens de l'innovation managériale, point central de son processus d'appropriation, conduit nécessairement à définir les trois éléments qui la composent : son substrat technique, sa philosophie gestionnaire et sa vision simplifiée des relations organisationnelles (Hatchuel et Weil, 1992), en généralisant ce schéma aux innovations managériales (David, 1996). Le substrat technique désigne l'ensemble des supports concrets sur lequel repose l'innovation et qui lui permet de fonctionner. Tableaux, courbes, graphiques ou encore interfaces d'outils informatiques en sont des exemples. Ce sont donc tous les éléments physiques, tangibles et visibles de l'innovation managériale pour les acteurs de l'organisation. Ils indiquent et guident la manière de réaliser les activités, les tâches en lien avec l'innovation et démontrent que l'innovation est bien mise en place (Mamman, 2009). La vision simplifiée des relations organisationnelles définit les rapports entre les acteurs de l'organisation influencés par l'outil parce qu'ils l'utilisent ou se conforment aux prescriptions de l'outil (Hatchuel et Weil, 1992). En d'autres termes, l'innovation managériale scénarise l'action d'un ensemble d'acteurs. A ce titre, elle modifie l'organisation et les rôles habituellement affectés aux acteurs (Alter, 2000). Enfin, la philosophie gestionnaire traduit l'esprit et les comportements de travail que l'innovation promeut. La philosophie est le reflet des hypothèses sous-jacentes à l'innovation sur la manière de manager une organisation ou de résoudre des problèmes organisationnels, elle constitue le cœur de l'innovation sur lequel les deux autres éléments sont construits (Mamman, 2009). Ainsi, l'innovation managériale passe par des "mythes rationnels" (Hatchuel et Weil, 1992), permettant de mobiliser les acteurs et favorisant le changement de perception (Segrestin, 2004).

Les travaux sur les composants des outils de gestion (Hatchuel et Weil, 1992) ou des innovations (David, 1996; Mamman, 2009) permettent de mieux appréhender ces objets en lien avec les dynamiques organisationnelles dans lesquelles ils sont intégrés. Il nous semble intéressant d'approfondir ces travaux sous l'angle de l'analyse de l'apport de chacun de ces composants (substrat technique, philosophie gestionnaire et vision simplifiée des relations organisationnelles), mais aussi leur interaction dans ce processus d'appropriation. En nous concentrant sur le processus d'innovation managériale à travers la question de l'appropriation, nous proposons de mener une réflexion sur le rôle du substrat technique dans le succès de ce processus. Parce qu'il est l'élément concret qui matérialise l'innovation, il convient de s'interroger sur la manière dont il peut faciliter l'appropriation de l'innovation par les acteurs de l'organisation. En effet, «la bonne parole gestionnaire n'a pas de vertus auto-réalisatrices : les meilleures idées ne sont efficaces que si elles sont outillées" (Segrestin, 2004, p. 24). La nature tangible du substrat technique permet l'actionnabilité totale ou partielle de l'innovation managériale. A contrario, la philosophie gestionnaire, cœur de l'innovation managériale, est un élément intangible particulièrement difficile à saisir pour les acteurs de l'organisation. Mamman (2009) rappelle que les difficultés liées à la bonne appropriation de cette philosophie peuvent être de plusieurs natures. Du point de vue des utilisateurs, la philosophie peut être mal partagée ou encore mal comprise. Les concepteurs peuvent également avoir des difficultés à la transmettre, à la présenter explicitement et à la communiquer. Cela interroge donc le rôle du substrat technique comme support de l'interaction entre l'intention des concepteurs et la compréhension des utilisateurs, puisqu'il est le support tangible et concret de l'innovation managériale. Cette interrogation sur le substrat technique peut se justifier au regard des travaux qui rappellent la nécessité de réintroduire le matériel dans l'analyse de l'activité organisationnelle (Orlikowski et Scott, 2008; Orlikowski, 2010; Leonardi, 2013). En effet, les aspects matériels font partie intégrante des activités et relations quotidiennes mais sont rarement analysés (Orlikowski et Scott, 2008). Dans la mesure où l'aspect matériel reflète et affecte le système social dans lequel il est encastré (Orlikowski, 1992), l'analyse de la définition des caractéristiques du substrat technique et ses évolutions permettent de comprendre la manière dont se négocie le sens de l'innovation entre les acteurs et avec les concepteurs. En effet, les acteurs conçoivent et adoptent des technologies avec des objectifs donnés qui se retrouvent «encodés» dans les objets (Orlikowski, 1992). Ainsi, il est possible de mobiliser la perspective de «l'appropriation » dans les travaux sur la sociomatérialité (Leonardi et Barley, 2010). Cette perspective considère que les concepteurs des technologies ont une image de la manière dont celle-ci doit être utilisée, mais que leur usage peut se faire de manière conforme ou non par rapport à ce qui avait été imaginé. Cette image de l'utilisation concerne tant les caractéristiques formelles de la technologie que son "esprit» (DeSanctis et Poole, 1994). Les managers doivent ainsi favoriser un espace de liberté dans l'utilisation de l'innovation tout en s'assurant que celle-ci ne dérive pas trop de l'intention initiale (Ansari et al., 2014), et favoriser un équilibre entre standardisation et adaptation (Alter, 2000; Ansari et al., 2014). A ce titre, la mobilisation des travaux sur la sociomatérialité permet de mener une analyse de l'appropriation en cherchant à comprendre comment les concepteurs construisent des contraintes physiques pour favoriser une utilisation particulière de l'innovation (Poole et DeSanctis, 1990), même si les acteurs restent libres d'en modifier les caractéristiques (Orlikoswki, 1992). Ainsi, la dimension matérielle peut 
contraindre la conception à l'usage de l'innovation (Lorino, 2002) qui est aux mains de l'ensemble des acteurs impliqués dans le processus. De plus, cette réflexion sur le rôle du substrat technique implique d'analyser les possibles interactions entre substrat technique et les autres composants de l'innovation au cours de sa construction. Les aspects du processus interprétatif sont particulièrement intéressants au cours de l'appropriation et la construction de sens de l'innovation implique l'ensemble de ses constituants. Eclairer le rôle du substrat technique comme médiateur entre intention des concepteurs et compréhension et intérêt des utilisateurs conduit à interroger l'interaction entre les différents éléments dans la construction de l'innovation. En effet, s'intéresser à la dimension matérielle ne doit pas aboutir à opposer social et matériel. Ces deux dimensions sont imbriquées et se contraignent mutuellement (Orlikowski et Scott, 2008). Orlikowski (1992) a ainsi démontré que la technologie, qui représente un substrat technique, s'apparente à un construit social résultant de l'action humaine et des propriétés structurelles du système organisationnel. Si l'appropriation implique l'interprétation par les acteurs de l'innovation managériale, et que celle-ci repose sur le substrat technique, alors il est intéressant de s'interroger sur la construction réciproque et progressive de ces éléments en interaction. En effet, la prise en compte de la dimension matérielle permet également de mieux appréhender la manière dont les acteurs utilisent l'innovation, à travers l'évolution de leurs pratiques de travail, en s'inscrivant dans la perspective de l'«enactment ", (Leonardi et Barley, 2010). La dimension matérielle est le support de l'action des acteurs. Au fil de son utilisation, et parce qu'il permet aux acteurs de résoudre les situations problématiques auxquels ils sont confrontés, le substrat technique va donner du sens aux situations et concourir à l'émergence de la structure sociale associée (Leonardi et Barley, 2010).

\section{Méthodologie}

Compte tenu de la perspective exploratoire de notre recherche et de la complexité des deux processus imbriqués analysés, l'innovation managériale et l'appropriation, nous avons fait le choix de réaliser une étude de cas unique (Eisenhardt, 1989; Yin, 1989). Le cas choisi nous permet de réaliser une analyse longitudinale dans une perspective processuelle afin de comprendre de quelle manière et pourquoi les choses apparaissent, se développent, évoluent ou encore disparaissent (Langley et al., 2013). Cette perspective permet de décrire à la fois le contexte, le contenu et les éléments du processus qui expliquent son succès ou encore les difficultés rencontrées, et met en évidence sa nature politique, culturelle, interactive et non-linéaire (Stensaker et Langley, 2010). De plus, les modes d'investigation choisis nous permettent d'accéder aux pratiques concrètes d'appropriation de l'innovation étudiée. Or, c'est à travers ces pratiques que nous pouvons réellement comprendre les processus en question (Rouleau et al., 2007).

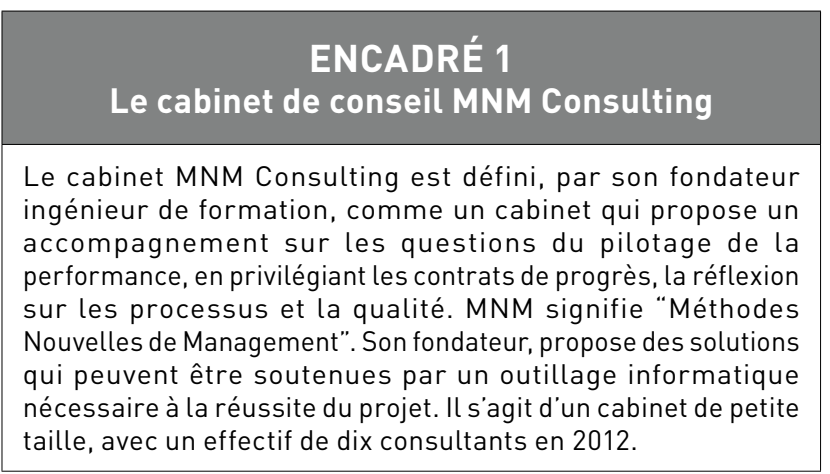

\section{Présentation de l'innovation managériale Chez VALEO : LA MÉTHODE 5 STEPS}

Nous étudions le cas de la méthode 5 steps, conçue au cours d'une collaboration entre un cabinet de conseil MNM Consulting (encadré 1) et le groupe Valeo, à l'initiative du Directeur des Systèmes d'Information (DSI) du Groupe. L'analyse de la conception et de l'évolution de cette innovation managériale entre 2005 et 2010 permet d'éclairer le processus d'innovation managériale de manière complète en nous concentrant sur les enjeux de l'appropriation:

La méthode 5 steps émerge au sein de Valeo pour répondre à des difficultés de mise en œuvre de la stratégie et d'écarts de maturité entre ses sites dispersés géographiquement (pays émergents, acquisitions, cessions d'entités, etc.). Cette méthode vise à piloter de manière harmonisée l'acquisition et le renouvellement des capacités organisationnelles dont chaque entité de l'organisation a besoin pour mettre en œuvre la stratégie. Elle repose sur des roadmaps de management qui sont des matrices à cinq niveaux de progrès qui décrivent la maturité des entités de l'organisation sur un sujet (cf. annexe 1). Cette méthode se distingue des méthodes de gestion de projet en décrivant non pas des tâches à réaliser dans le temps mais des évolutions de l'état et de la maturité des entités en dépassant les référentiels existants de type CMMI, ISO 9000 ou Cobit ${ }^{5}$. La méthode s'apparente pleinement à une innovation managériale : elle s'inscrit dans une volonté de marquer une rupture dans les pratiques existantes, en introduisant une dimension participative dans une organisation où la culture top-down est prégnante et très marquée. Son caractère innovant réside dans la combinaison et l'application des logiques de progression, d'apprentissage et de pilotage à l'acquisition des capacités organisationnelles, et se distingue à ce titre d'autres outils ou dispositifs. Tout d'abord, la méthode 5 steps poursuit une volonté commune au management par objectifs et dispositifs de tableaux de bord : la déclinaison de la stratégie le long de la ligne hiérarchique, avec l'alignement des actions quotidiennes avec la stratégie. De plus, elle suit la même logique de déclinaison en unités de "mesure » qui assure la mise en action mais également le pilotage. Elle mêle ensuite à ces démarches l'ambition des méthodes qui relèvent du mouvement de la qualité, dans lequel l'idéal poursuivi est celui de l'amélioration continue et elle passe pour cela par une mesure de la maturité des processus en cinq niveaux (cf. annexe 1). Elle vise une opérationnalisation de ces capacités par

5. Référentiels qui visent à appréhender, mesurer et améliorer les pratiques des entreprises, que ce soit dans les processus d’ingénierie ou les processus informatiques, notamment à partir d'indicateurs de progrès. 
le déploiement de roadmaps, dont l'unité de base est un livrable, preuve matérielle de la progression dans la grille de maturité.

Nous analysons la conception et l'évolution de cette méthode entre 2005 et 2010 . Nous retrouvons dans cette période les différentes phases spécifiques au processus d'innovation managériale, que nous abordons sous l'angle de l'appropriation. Les acteurs testent leur nouvelle méthode, la font évoluer au fil de sa mise en œuvre. Cette méthode fait l'objet d'un grand effort de théorisation dans le cadre d'un projet de recherche (encadré 2) qui conduit également à faire évoluer le contenu de la méthode. Un composant de cette innovation managériale est particulièrement travaillé : le SI qui supporte sa mise en œuvre et qui évolue au cours de la période. Un premier SI est utilisé lors de la conception initiale de la méthode, puis un second SI spécifique est conçu pour mieux répondre aux besoins de mise en œuvre de la méthode.

\section{LA COLLECTE DES DONNÉES EMPIRIQUES}

Notre recherche vise à comprendre le processus d'appropriation au cours de la construction d'une innovation managériale, en nous focalisant sur le rôle que peut jouer le substrat technique dans cette appropriation, et notamment dans l'interaction entre innovation et acteurs de l'organisation. Cette démarche processuelle implique de récolter des données longitudinales pour pouvoir comprendre comment un processus se déroule dans le temps (Langley et al., 2013). Au cours de notre étude, les matériaux ont varié du fait de différents accès au terrain. Ils nous ont permis d'analyser trois temps forts de ce processus d'innovation managériale : l'invention initiale au sein de Valeo, le travail de théorisation et de construction du SI au cours du projet de recherche ANR et la relance de la dynamique de mise en œuvre suite à l'essoufflement de l'utilisation de la méthode. Nos interactions avec le cabinet MNM Consulting ont été particulièrement riches dans la période analysée, notamment à travers la participation au projet de recherche Pilot 2.0 et une recherche plus générale sur les innovations managériales qui a impliqué une méthodologie d'observation participante entre septembre 2008 et avril 2010. La richesse des matériaux, la multiplicité des acteurs interviewés et l'analyse de nombreux documents permettent la triangulation des données et limitent au maximum le biais de rationalisation a posteriori. Notre méthode de recueil de données mixe donc des entretiens semi-directifs, de l'observation participante et de l'analyse de documents et d'archives (Tableau 1 - Données empiriques).

\section{MÉTHOdE D'ANALYSE}

La problématique de recherche qui vise à éclairer le rôle du substrat technique dans l'interaction entre l'innovation managériale et les acteurs positionne le questionnement dans une recherche compréhensive car nous nous intéressons aux acteurs, à leurs motivations, à la façon dont ils agissent et interagissent (Dumez, 2013). La durée du terrain et la nature de la recherche nous ont conduits à exploiter un matériau empirique riche mais hétérogène. Comme le soulignent Langley et al. (2013), afin de développer une compréhension fine des processus à un niveau micro, il est nécessaire d'avoir un engagement profond et prolongé combiné à une attention et une rigueur analytique.

\begin{tabular}{l} 
ENCADRÉ 2 \\
Présentation du projet ANR Pilot 2.0 \\
\hline Le projet PILOT 2.0, financé par l'Agence Nationale de la \\
Recherche (ANR), vise à construire, à destination des \\
organisations étendues, des méthodes et des outils robustes \\
et efficaces permettant la mise sous contrôle des capacités \\
organisationnelles et le pilotage des plans de progrès. Il vise \\
plus particulièrement le développement d'une plateforme sur \\
des technologies web, à base de logiciels libres et de composants \\
standardisés, capable de supporter la complexité de la réalité \\
des entreprises étendues et le volume important des mesures \\
en cause et d'offrir une interface conviviale et des fonctions \\
collaboratives. Avec une vision «pilotage 2.0», les opérationnels \\
collaborent en réseaux virtuels, adaptent les outils à leur besoin \\
local et deviennent de véritables acteurs du pilotage. Ce projet \\
coordonné par MNM Consulting implique plusieurs partenaires: \\
Valeo, le Conseil Général du Vaucluse, les équipes de recherche \\
IRCCYN de l'Ecole Centrale de Nantes et M-Lab de l'Université \\
Paris-Dauphine. L'objectif de ce financement était de déterminer \\
quelles étaient les possibilités d'évolution du SI dans une logique \\
«2.0» afin de faciliter l'appropriation des roadmaps de \\
management diffusées via le SI de l'époque.
\end{tabular}

Nous avons donc cherché à organiser et mettre en forme notre matériau en série selon les trois grandes périodes d'analyse. Nous avons ainsi pu faire un traçage de processus en variant les données (George et Benett, 2005). La mise en forme des données s'est faite au moyen de deux templates (Annexe 2) afin de permettre la création de séries de données comparables entre elles pour un traitement analytique des données, l'objectif étant d'éviter le risque de circularité (Dumez, 2013). Nous avons construit un premier tableau qui nous a permis pour chaque occasion de recueil de matériaux d'identifier des verbatims saillants et de faire émerger des premiers commentaires, analyses et thèmes. Nous avons réalisé un second template afin de comprendre le rôle du substrat technique dans l'appropriation puisque nous considérions que l'analyse de ses caractéristiques et de ses évolutions permettait de comprendre la manière dont se négocie entre les acteurs le sens de l'innovation. Celui-ci visait, à travers les trois périodes à révéler les évolutions de la définition du SI à travers ses propriétés et ses fonctionnalités et à les mettre en regard de la définition de la méthode. Nous avons systématiquement collecté au moins 3 types de sources de données à chacune des phases afin de pouvoir faire une triangulation. Cette analyse de l'appropriation en 3 périodes permet une décomposition du processus et permet donc une logique de réplication longitudinale (Langley et al., 2013); au sein de ces données longitudinales, nous avons identifié des unités d'analyse comparables. Ces templates permettent de ranger les données en opérant des rapprochements qui n'auraient pas été faits spontanément par les chercheurs et viennent donc enrichir le travail analytique (Dumez, 2013).

\section{Résultats de l'étude de cas}

\section{TROIS PHASES DISTINCTES DANS LA CONSTRUCTION ET L'APPROPRIATION DE L'INNOVATION MANAGÉRIALE}

L'analyse longitudinale de ce cas nous permet d'étudier à travers la question de l'appropriation, l'évolution du sens et du contenu de l'innovation managériale. Notre cas révèle une place particulière dans ce processus accordée à la définition du SI qui soutient la 


\begin{tabular}{|c|c|c|}
\hline \multicolumn{3}{|c|}{$\begin{array}{c}\text { TABLEAU } 1 \\
\text { Données empiriques }\end{array}$} \\
\hline Dates & Modalités de collecte & Objectifs \\
\hline \multicolumn{2}{|c|}{$\begin{array}{l}\text { Entretiens semi-directifs } \\
\text { (Retranscrits sous forme de notes de synthèse) }\end{array}$} & $\begin{array}{l}\text { Confronter les points de vue des différents acteurs et comprendre dans } \\
\text { le temps la construction de l'innovation managériale et le processus } \\
\text { d'appropriation }\end{array}$ \\
\hline 2006 & $\begin{array}{l}2 \text { entretiens avec les concepteurs de } \\
\text { la méthode }\end{array}$ & $\begin{array}{l}\text { Comprendre les raisons et le contexte de conception de la méthode } \\
\text { au sein de Valeo et appréhender leur vision de la méthode et la manière } \\
\text { de la mettre en œuvre au sein de l'organisation }\end{array}$ \\
\hline 2008 & $\begin{array}{l}47 \text { entretiens auprès des utilisateurs de la } \\
\text { méthode au sein de Valeo }{ }^{6} \text { (rédacteurs de } \\
\text { roadmaps, auditeurs, responsables d'axes et } \\
\text { ingénieurs opérationnels) }\end{array}$ & $\begin{array}{l}\text { Recueillir l'avis des utilisateurs et mesurer l'impact de son utilisation } \\
\text { sur l'organisation }\end{array}$ \\
\hline $\begin{array}{l}2006 \text { et } \\
2010\end{array}$ & $\begin{array}{l}2 \text { entretiens avec le directeur de l'audit } \\
\text { interne de Valeo }\end{array}$ & Recueillir des données sur l'utilisation et impacts sur l'organisation \\
\hline 2008 & 2 entretiens avec 2 consultant & Confronter le point de vue des consultants à ceux des concepteurs \\
\hline 2009 & $\begin{array}{l}2 \text { entretiens avec les concepteurs de } \\
\text { la méthode }\end{array}$ & $\begin{array}{l}\text { Aborder le bilan sur l'utilisation de la méthode au sein de Valeo, les } \\
\text { raisons des besoins d'évolution mais aussi le contenu de ses évolutions, }\end{array}$ \\
\hline 2011 & $\begin{array}{l}1 \text { entretien avec le directeur du cabinet } \\
\text { de conseil }\end{array}$ & $\begin{array}{l}\text { Comprendre la relance de la dynamique de la méthode soutenue par } \\
\text { le nouvel SI au sein de Valeo }\end{array}$ \\
\hline 2011 & 3 entretiens avec 2 consultants & $\begin{array}{l}\text { Comprendre leur vision de la méthode, la réalité de sa mise en œuvre, } \\
\text { les difficultés rencontrées }\end{array}$ \\
\hline \multicolumn{2}{|c|}{$\begin{array}{l}\text { Observation participante } \\
\text { (prise de notes systématique et carnet de bord) }\end{array}$} & $\begin{array}{l}\text { Analyser les interactions entre MNM et Valeo et interpréter les } \\
\text { problématiques d'appropriation du SI }\end{array}$ \\
\hline $\begin{array}{l}2008 / \\
2010\end{array}$ & $\begin{array}{l}\text { Projet de recherche ANR : } \\
\text { - } 11 \text { réunions chez MNM Consulting } \\
\text { - } 2 \text { réunions chez Valeo } \\
\text { - } 1 \text { réunion participants projets ANR } \\
\text { - Formation à la méthode par des consultants }\end{array}$ & $\begin{array}{l}\text { Les réunions portaient sur la conception du nouveau SI pour soutenir la } \\
\text { méthode } 5 \text { steps. Elles nous ont également permis de recueillir la vision } \\
\text { de la méthode par ses concepteurs. }\end{array}$ \\
\hline $\begin{array}{l}2009 / \\
2012\end{array}$ & $\begin{array}{l}\text { Recherche sur les innovations managériales } \\
\text { (présence } 2 \text { à } 3 \text { jours par semaine au sein du } \\
\text { cabinet de conseil) : } \\
-2 \text { réunions avec MNM et Valeo } \\
\text { - } 11 \text { réunions chez MNM } \\
\text { - échanges informels avec les concepteurs et } \\
\quad \text { les consultants }\end{array}$ & $\begin{array}{l}\text { Les réunions entre MNM et Valeo visent à définir les modalités de relance } \\
\text { de la méthode, les sujets concernés, la manière de former les utilisateurs. } \\
\text { Au cours des réunions au sein de MNM, la situation de Valeo est évoquée } \\
\text { entre consultants. Ces réunions sont l'occasion d'aborder les difficultés } \\
\text { rencontrées, l'acceptation et la compréhension de la méthode par les } \\
\text { utilisateurs au sein de Valeo } \\
\text { Les échanges informels fréquents permettent d'apporter ainsi une } \\
\text { compréhension fine des évènements }\end{array}$ \\
\hline \multicolumn{2}{|c|}{ Analyse de documents } & $\begin{array}{l}\text { Trianguler les interprétations des interactions entre les acteurs avec } \\
\text { des données secondaires pour un objectif confirmatoire }\end{array}$ \\
\hline \multicolumn{2}{|c|}{$\begin{array}{l}\text { - supports de formation, guides utilisateurs des deux SI, } \\
\text { supports de communication sur la méthode } \\
\text { - accès aux plateformes informatiques }\end{array}$} & $\begin{array}{l}\text { Repérer le vocabulaire utilisé et les idées véhiculées dans ces supports } \\
\text { en lien avec l'innovation managériale } \\
\text { Recueillir des informations sur les utilisations des deux SI dans le temps }\end{array}$ \\
\hline
\end{tabular}

mise en œuvre de la méthode. Cette analyse processuelle met en évidence le rôle du substrat technique dans l'appropriation via le rôle qu'il joue dans la construction du sens partagé entre les différents acteurs impliqués dans ce processus. Nous observons trois grandes périodes dans l'étude de ce cas. Entre 2005 et 2009, nous assistons à la conception et mise en œuvre d'une méthode, un temps intégrée aux pratiques de l'organisation, mais qui finit par être abandonnée en 2009. Entre 2008 et 2010, nous entrons dans une phase davantage réflexive d'analyse et d'amélioration du dispositif en place. Dans le cadre du projet de recherche ANR Pilot 2.0, un SI dédié est conçu : Roadmap Manager. Suite à la quasi-disparition de la méthode au sein de
Valeo, le cabinet de conseil est à nouveau sollicité pour relancer une dynamique d'utilisation de la méthode au sein de Valeo soutenue cette fois par Roadmap Manager. La figure 1 synthétise les moments clés du processus d'appropriation de la méthode 5 steps et de l'évolution du rôle du SI par les concepteurs.

\section{Période 1 : Conception et mise en œuvre de l'innovation managériale}

Entre 2005 et 2009, nous assistons à la conception et mise en œuvre d'une méthode pour répondre à une situation problématique identifiée par le DSI du groupe Valeo. En 2004, le DSI fait appel à la société MNM Consulting pour résoudre des problèmes de

6. Ces entretiens ont été menés par Ibrahima Fall dans le cadre de sa thèse de doctorat : Approche «gestionnaire de la capacité organisationnelle et pilotage du progrès : Apports d'un dispositif pionnier de gestion des capacités organisationnelles dans une entreprise mondialisée, soutenue en 2008 à l'Ecole des Mines de Paris; et Philippe Rauffet dans le cadre de sa thèse de doctorat: Prise en compte des facteurs formels et contextuels dans la gestion des capacités organisationnelles. Application aux organisations matricielles, soutenue en 2010 à l'Ecole Centrale de Nantes. 
FIGURE 1

Chronologie du processus d'innovation managériale pour la méthode 5 steps

Période 1 : Conception et mise en oeuvre de l'innovation managériale

Période 2 : Analyse du dispositif et conception d'un SI dédié

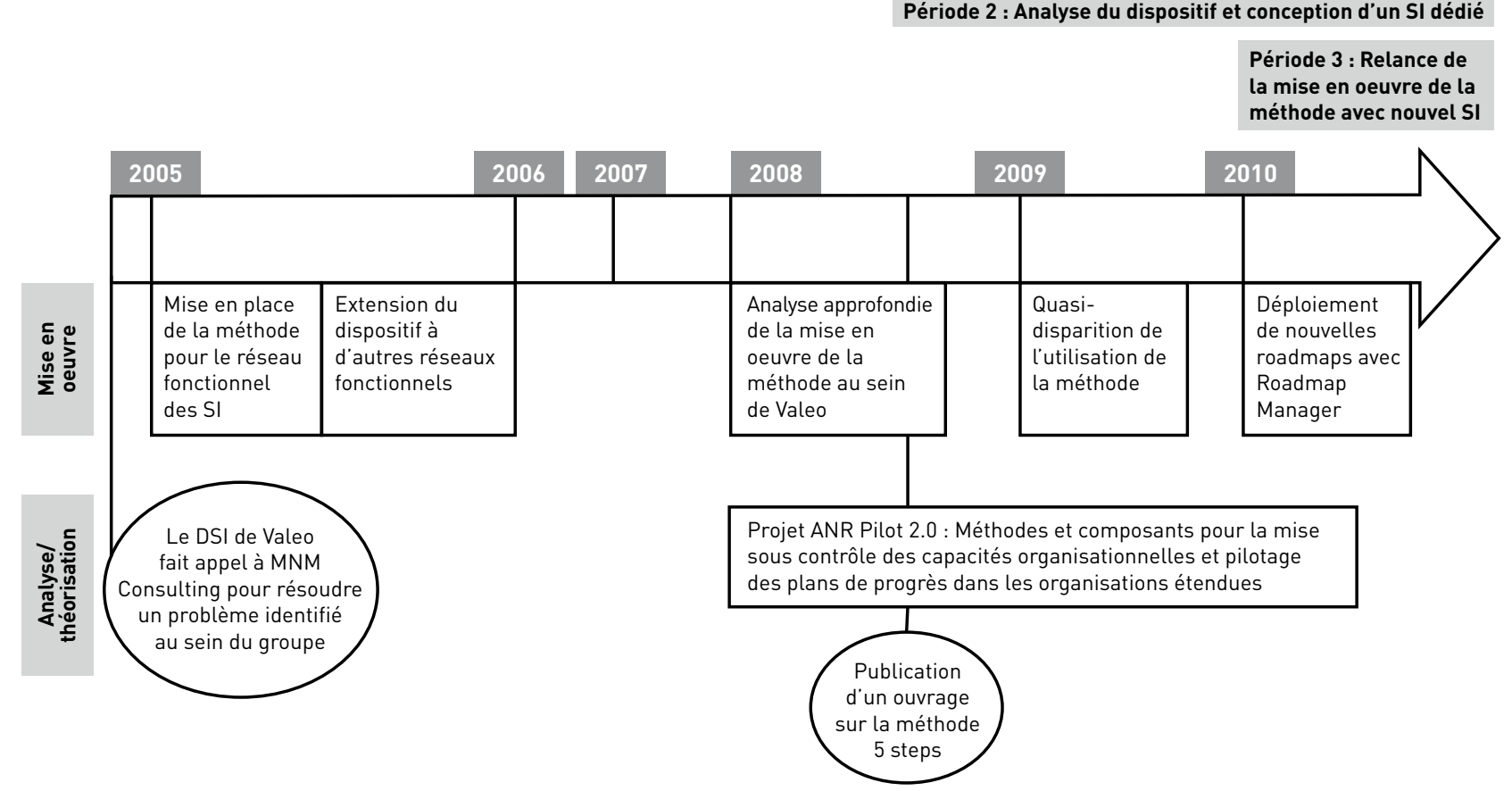

mise en œuvre de la stratégie et d'écarts de maturité entre ses sites situés dans différents pays. La performance organisationnelle de Valeo repose sur un équilibre entre l'autonomie et la standardisation : les entités opérationnelles sont autonomes pour mettre en œuvre la stratégie du groupe mais doivent respecter un certain nombre de procédures et de standards formalisés par la direction générale. Valeo souhaite déployer un dispositif permettant de décrire la progression des sites, quant à leur acquisition des standards de l'entreprise et d'en avoir une vision claire. Le DSI et le directeur du cabinet de conseil MNM Consulting développent une méthode pour répondre à ces enjeux. Ainsi, au premier semestre 2005, des roadmaps sont rédigées et déployées pour le réseau fonctionnel du SI, puis cette solution est étendue à d'autres réseaux fonctionnels, au second semestre 2005. Plus de 80 roadmaps sont alors rédigées et déployées dans plus de 200 entités organisationnelles du groupe (soit 6 réseaux fonctionnels et 9 branches produits). Ce déploiement s'appuie sur une utilisation et configuration d'éléments du SI existant (Matrix avec comme support un ERP SAP). En effet, les concepteurs se sont vus rapidement confrontés au problème de déploiement de l'innovation managériale dans une entreprise étendue comme Valeo. En l'absence d'une solution existante chez les éditeurs informatiques, les développeurs ont adapté le SI de Valeo aux propriétés de la méthode 5 steps $^{7}$. En 2008, plus de 2000 personnes ont accès à cette plateforme informatique, concrétisation matérielle majeure de la méthode au sein de Valeo. Ce déploiement rapide dans toute l'organisation est possible du fait de la culture de Valeo : «Grâce à la culture Valeo qui est forte : on ne remet pas souvent en question une méthodologie standard groupe.» (Directeur audit Interne Valeo, 2006).

La conception de cette innovation s'appuie sur un audit d'outil existant mais peu utilisé. L'ambition est de le transformer et de marquer une rupture avec les pratiques existantes : «Précédemment, le questionnaire était un questionnaire d'audit, il était peu utilisé par les sites [...] la grosse différence pour moi aujourd'hui est que notre référentiel n'est plus un référentiel d'audit mais un outil de progrès pour les sites», "c'est un vraie rupture par rapport aux méthodes d'audit classiques, comme l'ISO 9000» (Directeur audit Interne Valeo, 2006). La méthode se pose également en rupture de la culture top-down existante : les roadmaps sont vus comme « un élément essentiel du management car elles servent la capitalisation du savoir-faire» (DSI, 2006). Elles doivent servir à «mobiliser les équipes» et "s'inscrivent dans une logique d'amélioration continue dans une démarche participative» (Dirigeant de MNM Consulting, 2006). Cette ambition est clairement assumée : «cela ne sert à rien de faire des roadmaps si les gens ne se l'approprient pas comme outil de management» (DSI Valeo, 2006).

Cependant l'analyse menée en $2008^{8}$ met en évidence une appropriation incomplète de l'innovation par les utilisateurs et notamment par les opérationnels. En effet, la méthode ne parvient pas à s'inscrire dans les routines de l'organisation et finit par ne plus être utilisée. La difficulté majeure réside dans

7. Son adaptation par les développeurs de MNM Consulting a été rapide et a représenté 50 jours de travail.

8. Cette analyse a été menée par deux doctorants Ibrahima Fall et Philippe Rauffet dans le cadre de leur thèse de doctorat. 
l'incompréhension de la philosophie de la méthode par les opérationnels, qui la perçoivent essentiellement comme un outil de reporting au service des directions fonctionnelles, et non comme méthode de soutien à leur progression et apprentissage sur les sujets traités par les roadmaps («il y a la méthode qui devrait nous permettre de situer notre progression sur chacun des domaines et de s'assurer qu'on est sur la bonne voie, mais finalement Matrix c'est juste un outil de reporting qui sert à remonter les informations au groupe», ingénieur opérationnel). L'innovation n'est pas utilisée comme un outil de management, tel que pensé par ses concepteurs, mais dans sa fonction plus restrictive de reporting et pilotage. Le manque d'ergonomie et l'interface sommaire rendent difficile l'utilisation de la roadmap («Matrix est un "outil à la russe», c'est un outil rustique qui marche mais il est assez complexe, avec des soucis d'interface», développeur MNM Consulting). En conséquence, de nombreux opérationnels ont pris l'habitude de la charger en format excel pour la lire et s'évaluer. Cela n'affecte pas leur compréhension et leur intérêt pour le contenu de la roadmap dans son rôle d'accompagnement du progrès mais réduit l'incitation à son utilisation régulière. De plus, l'absence de fonctionnalités de communication entre le rédacteur de la roadmap et les opérationnels pose des difficultés d'interprétation au niveau des pratiques à mettre en œuvre pour passer d'un niveau à un autre sur certaines roadmaps. Ainsi, les utilisateurs se retrouvent dans une situation d'incompréhension de la méthode et d'incapacité à l'utiliser («le problème c'est que je ne sais pas à qui m’adresser quand je ne comprends pas un livrable, et cela arrive souvent », opérationnel). L'accompagnement du progrès voulu par les concepteurs n'est pas réellement permis par le SI. Cette situation montre que la philosophie gestionnaire liée à la méthode n'est pas diffusée telle que pensée par les concepteurs. La dimension de management du progrès associée à une logique participative de l'amélioration continue n'est pas comprise et perçue par les utilisateurs. Cette réduction du sens auprès des opérationnels limite leur intérêt pour la méthode. En 2009, le directeur du cabinet de conseil reconnaît que la méthode n'est plus réellement mise en œuvre au sein de Valeo.

\section{Période 2 : Conception d'un SI dédié}

Entre 2008 et 2010, nous entrons dans une phase réflexive d'analyse et d'amélioration du dispositif en place. Les analyses menées auprès des utilisateurs ont mis en lumière les grandes difficultés d'appropriation de la méthode. Le projet de recherche ANR Pilot 2.0 a vocation à proposer des méthodes et composants pour la mise sous contrôle des capacités organisationnelles, afin de permettre le pilotage des plans de progrès dans les organisations étendues. Cette réflexion conduit à l'étiquetage de la méthode déployée au sein de Valeo sous le nom de «méthode 5 steps »". Le projet de recherche a pour principal résultat opérationnel le développement d'un SI dédié à la méthode, appelé Roadmap Manager. Nous assistons donc à la redéfinition substantielle du substrat technique qui matérialise l'innovation. Le SI est alors perçu comme un levier d'actionnabilité mais également d'évolution de la méthode 5 steps.

L'objectif des concepteurs est que les opérationnels et les managers s'emparent de la méthode comme un outil de progrès qui s'appuie sur une logique de dialogue entre acteurs (entre les entités, avec la hiérarchie), dans une perspective d'amélioration continue. Roadmap Manager inclut de nouvelles fonctionnalités et des améliorations qui ont vocation à faciliter l'utilisation de la méthode 5 steps via les propriétés intrinsèques du SI (accessibilité des données, paramétrage des menus, interface modulable, etc.). Une attention particulière est consacrée à l'ergonomie de la plateforme pour faciliter la lecture de la roadmap et l'auto-évaluation par les opérationnels. Par exemple, des fonctions de feedback sont incluses pour assurer le dialogue et les échanges au sujet de la roadmap entre les différents acteurs impliqués dans la démarche (opérationnels, rédacteurs, fonctionnels, directions). Ces options ont plusieurs objectifs. Tout d'abord, elles doivent permettre un dialogue entre les utilisateurs de la roadmap et les rédacteurs, pour favoriser la compréhension du contenu de la roadmap par les utilisateurs. De plus, elles doivent être l'occasion de récolter des bonnes pratiques et des suggestions d'amélioration de la part des utilisateurs dans une logique où de nouvelles versions de roadmaps sont rédigées régulièrement dans une démarche d'amélioration continue. Les utilisateurs sont ainsi dans une position de partie prenante de l'évolution de la méthode et de co-concepteurs du processus car ces évolutions sur le substrat technique proviennent des remontées des acteurs interrogés dans la première phase. Une réflexion est également menée sur les différents profils d'utilisateur (utilisateurs, rédacteurs, managers) et les informations spécifiques à chacun à faire apparaître sur l'interface du SI. Il s'agit de limiter l'affichage à l'information utile et ainsi faire passer plus simplement le message sur les objectifs de la méthode aux différents acteurs. Les fonctions de pilotage et les possibilités de création de tableaux de bord sont également améliorées.

\section{Période 3 : Relance de la dynamique d'utilisation de la méthode soutenue par le nouveau SI (Roadmap Manager)}

Au début de l'année 2010, le cabinet de conseil MNM Consulting est à nouveau sollicité pour relancer une dynamique d'utilisation de la méthode 5 steps au sein de Valeo soutenue cette fois par Roadmap Manager. La méthode est utilisée pour gérer des enjeux de la fonction "Risque, Assurance et Environnement» (RAE) du groupe. Il s'agit « d'un projet très important, vraiment stratégique, depuis une dizaine années, c'est une méthode sur laquelle je compte» (Directeur de la fonction RAE, qui a initié la relance de la méthode). Le projet démarre en février, les roadmaps et le SI sont d'abord testés sur deux entités pilotes pendant deux mois, puis la méthode est généralisée à l'ensemble des entités concernées par la problématique (80 entités).

Le test auprès des deux entités pilotes est l'occasion de nombreux échanges entre les porteurs de projet au sein de Valeo et les consultants au sujet des fonctionnalités à retenir dans Roadmap Manager ou encore de la gestion des droits des utilisateurs, avec la mise en place des profils d'utilisateurs. Une attention particulière est portée aux fonctions de feedback existantes pour faciliter les interactions des opérationnels avec les fonctionnels et éviter toute difficulté de compréhension, et surtout pouvoir prendre en compte les suggestions d'amélioration. Par exemple, plusieurs décisions de paramétrages de la plateforme sont actées au cours de réunions en mars 2010 : «il est nécessaire 
de pouvoir ajouter des documents types dans la roadmap sur la plateforme pour guider les opérationnels dans l'avancement... [...] les messages de feedback doivent directement être envoyés par mail à la personne concernée en plus de l'envoi du message dans l'outil» (Responsable RAE).

De plus, la mise en œuvre de la méthode est couplée au travail des auditeurs de Valeo. Ces acteurs sont donc directement impliqués dans le déploiement de la méthode. Il est important de rappeler que la philosophie de l'audit au sein de Valeo s'inscrit dans une logique d'harmonisation des pratiques entre les entités au sein d'une organisation mondiale et éclatée, et est aussi l'occasion d'identifier les «best practices» au sein des entités du groupe pour les diffuser ensuite dans cette logique d'harmonisation. Ce couplage à l'outil d'audit et les fonctionnalités de Roadmap Manager permettent une réelle convergence. La métrique de progression fournie par les roadmaps (note sur 5 niveaux à partir de l'avancement de la roadmap) devient un élément audité et une base de discussion des pratiques entre auditeur et audités.

A la fin de notre recherche en 2010, les premiers retours des utilisateurs sur la plateforme semblent positifs, notamment au moment du déploiement du pilote. La simplicité et la bonne ergonomie de la plateforme sont soulignées par les utilisateurs, les fonctions de feedback ont été utilisées par $25 \%$ des opérationnels au cours du pilote, une dizaine de livrables ont été modifiés suite à ces retours ${ }^{10}$. La dimension collaborative semble donc pouvoir s'installer. Après plusieurs mois d'utilisation, le directeur de l'audit interne de Valeo confirme : «Il arrive maintenant que l'on ait un écart moindre entre le self assessment et ce que les auditeurs trouvent. Les gens ont compris que ce n'était pas faire des points pour faire des points mais progresser de manière robuste». Plusieurs utilisateurs déclarent aux consultants «apprécier cette nouvelle vision de l'accompagnement du progrès à travers les roadmaps ». On constate donc que l'amélioration du substrat technique permet de faciliter les fonctions de dialogue entre les catégories d'acteurs et de simplifier sa compréhension. Cela permet donc de mieux véhiculer la philosophie pensée par les concepteurs et d'améliorer la dimension interprétative du processus d'appropriation.

Cette analyse des trois phases distinctes de l'appropriation de la méthode soutenue par deux SI différents nous permet de mettre en évidence notre premier résultat sur le rôle central du substrat technique dans l'appropriation de l'innovation managériale, dans la mesure où il est le support d'interaction entre concepteurs et utilisateurs. En effet, le substrat technique est le principal support physique et formel auquel ont accès les différents utilisateurs et qui incarne la méthode 5 steps aux yeux des utilisateurs. Méthode et outil sont présentés aux acteurs comme formant un tout, notamment aux moments des formations à l'outil qui sont l'occasion de sensibiliser les acteurs aux principes de la méthode. Le SI est un support concret et tangible qui permet de leur donner corps et de les illustrer. Le SI facilite donc la transportabilité de l'innovation au sein des entités du groupe Valeo. Cependant, cette dimension matérielle ne suffit pas à favoriser l'appropriation de l'innovation managériale. En effet, l'analyse des deux périodes de mise en œuvre (période 1 et 3) révèle des niveaux d'appropriation différents, notamment parce que le substrat technique n'assure pas un processus interprétatif correct entre l'intention des concepteurs et la compréhension de la méthode par ses utilisateurs. Comme nous avons pu le constater dans la période 1 , l'innovation est davantage perçue comme outil de reporting, que comme méthode de management comme le souhaitaient ses concepteurs. Au contraire, dans la période 3 , les évolutions du SI concourent à l'amélioration de ces processus interprétatifs et assure ainsi une meilleure convergence entre intention des concepteurs et perception des utilisateurs. Le SI donne davantage à voir la méthode plus directement dans sa philosophie pensée par les concepteurs.

\section{APPROPRIATION D'UNE INNOVATION MANAGÉRIALE : UNE INTERACTION RÉCIPROQUE ENTRE SUBSTRAT TECHNIQUE ET PHILOSOPHIE GESTIONNAIRE}

Notre premier résultat permet de montrer le rôle que joue le substrat technique comme médiateur entre concepteurs et utilisateurs, et dans quelle mesure il favorise l'appropriation lorsqu'il permet de transmettre la philosophie promue par son concepteur dans la création de sens autour de l'innovation par les utilisateurs. Notre analyse nous a permis de montrer que dans la première phase, un découplage des composants de l'innovation managériale, entre le substrat technique et la philosophie gestionnaire explique l'échec de l'appropriation de l'innovation managériale. Ce couplage des composants s'est fait dans un second temps, permettant ainsi un meilleur processus interprétatif par les acteurs en phase avec l'intention des concepteurs. Nous analysons donc maintenant dans quelle mesure la redéfinition du substrat technique a été tirée par la volonté de mieux traduire la philosophie gestionnaire. Notre analyse nous conduit à mettre en évidence une interaction réciproque entre substrat technique et philosophique gestionnaire dans la (re)construction de l'innovation pour une meilleure appropriation. Il est intéressant de noter que cette interaction se fait dans le temps et en fonction des acteurs impliqués dans la définition de l'innovation.

Tout d'abord, la seconde période (2008-2010) est l'occasion de capitaliser sur l'expérience concrète de mise en œuvre de la méthode au sein du groupe Valeo. Cette prise de recul conduit notamment à concevoir un SI dédié à la méthode afin d'améliorer son appropriation. Cette intention est clairement affichée au sein de Valeo ( Il s'agit d'une remontée sur le besoin de voir les objectifs globaux : vers un outil de management, vers un déploiement plus souple, vers un référentiel plus dynamique, vers une meilleure lisibilité des résultats, vers un outil plus ergonomique», chef de projet, Valeo), mais aussi dans l'équipe de consultants et développeurs de MNM Consulting ( LL'idée est de faire quelque chose de plus simple», "Les aspects collaboratifs seront plus développés", développeur).

L'analyse de la mise en œuvre réelle de la méthode et ses principes dans l'organisation conduit les concepteurs à faire évoluer une partie de son substrat technique afin qu'il puisse faciliter la compréhension et la mise en place effective de la philosophie gestionnaire pensée. L'objectif des concepteurs est que les opérationnels et les managers s'emparent de la méthode 
comme un outil de progrès qui s'appuie sur une logique de dialogue entre acteurs (entre les entités, avec la hiérarchie). Cette logique a guidé la construction du nouveau SI avec l'apparition des nouvelles fonctionnalités que nous avons décrites plus haut. Ainsi, le substrat technique joue un rôle important dans le processus interprétatif et supporte la construction du sens et la représentation partagée de l'innovation par les acteurs. Réciproquement, la conception du SI dédié participe au prolongement de la réflexion sur la définition et le contenu de la méthode par ces concepteurs. L'innovation continue à être précisée à travers la conception du SI, le développement de certaines fonctionnalités venant soutenir l'affirmation de principes qui composent l'innovation. On peut donc considérer que la conception du SI qui soutient la mise en œuvre de l'innovation managériale participe à l'achèvement de l'invention de l'innovation parce qu'elle nécessite de penser très finement des règles ou des principes qui vont venir composer l'innovation (règles d'animation du SI, gestion des droits des utilisateurs, paramétrages possibles du coté utilisateur, manuel d'utilisation, etc.). Ces règles ou principes permettent également de mieux traduire le sens de la philosophie gestionnaire des concepteurs. Par exemple, la conception du SI et de son paramétrage a conduit à s'interroger sur les possibilités d'accès aux résultats d'autoévaluation des entités sur les roadmaps. D’un point de vue technique, il n'y a pas de difficultés particulières à ce que chaque entité puisse consulter les résultats de toutes les autres entités du groupe, ou au contraire, que les résultats ne soient accessibles qu'aux seuls managers. Ces possibilités techniques conduisent les deux concepteurs à s'interroger sur l'utilisation de l'outil et la philosophie promue en fonction du choix technique réalisé.

L'accès aux résultats des autres entités peut en effet être utilisé comme moyen de générer du dialogue et de l'échange de bonnes pratiques entre entités, ou bien de stimuler une forme de concurrence interne entre les entités par rapport aux standards de performance qui sont fixés par la direction. Cela conduit donc à préciser encore la philosophie pensée par les concepteurs à travers cette formalisation technique.

L'interaction réciproque entre substrat technique et philosophie gestionnaire s'inscrit dans une volonté d'alignement entre l'intention des concepteurs et la perception des utilisateurs. Elle est le résultat d'un effort de réflexivité et d'apprentissage par les concepteurs qui prennent conscience progressivement des écarts qui existent entre ces deux dimensions. A ce titre, l'évolution du discours du directeur du cabinet de conseil est très révélatrice. La comparaison des présentations commerciales de la méthode par le directeur du cabinet en 2007 et 2010, ainsi que l'ouvrage sur la méthode publié en 2008, met en évidence l'importance relative grandissante de l'occurrence des mots clés relatifs à une vision de la méthode comme outil de management dans une démarche collaborative («management, stratégie, progrès, progressions, collaboration, ou encore capitalisation»). Nous constatons aussi la disparition progressive de termes comme le «reporting» ou encore les «standards» qui traduisent la disparition de la prégnance du vocabulaire classique dans la culture Valeo, au profit du vocabulaire spécifique aux objectifs affirmés de la méthode par son concepteur.

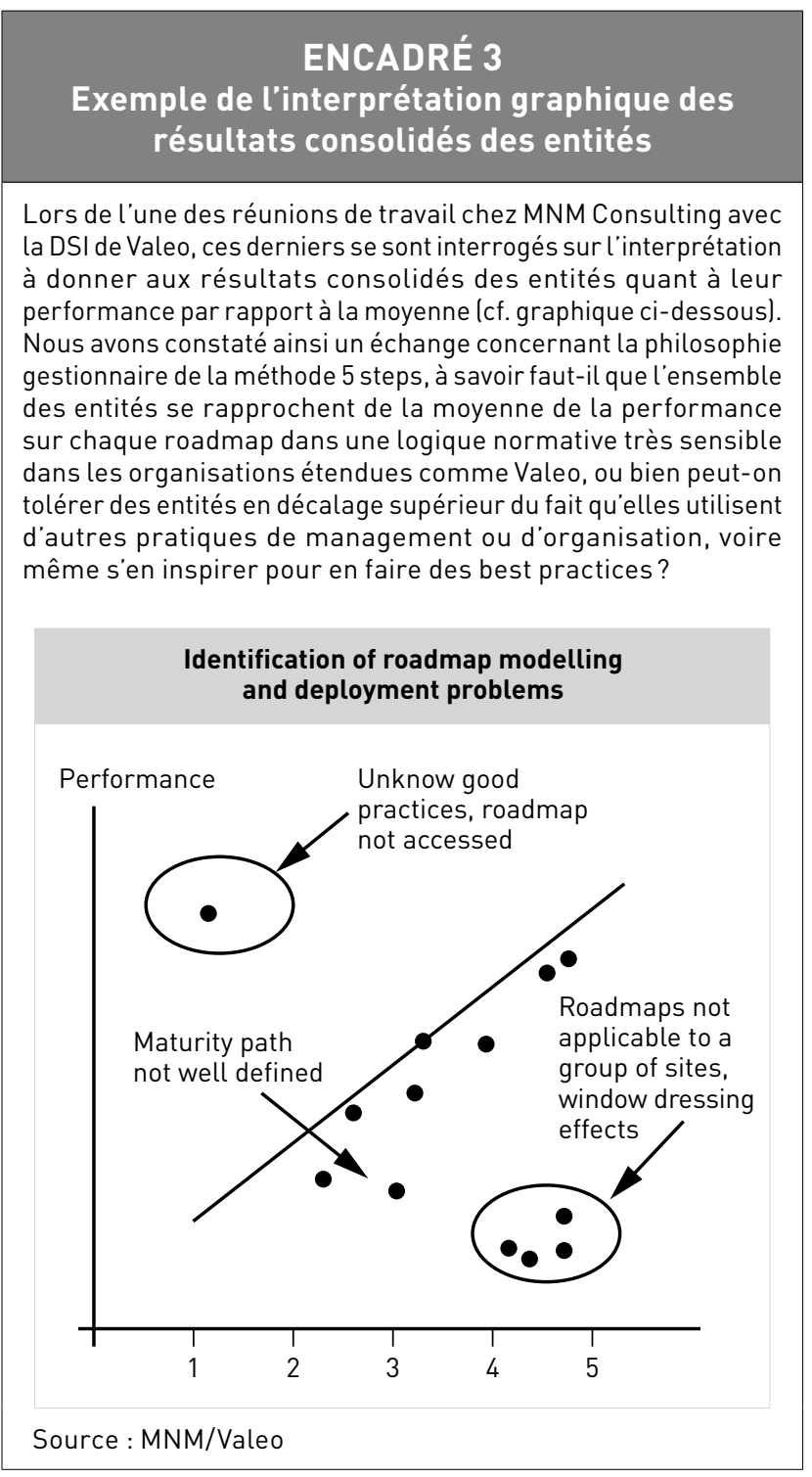

\section{APPROPRIATION D'UNE INNOVATION MANAGÉRIALE : L'ÉMERGENCE DE NOUVEAUX ACTEURS CLÉS QUI FAVORISENT L'APPROPRIATION}

L'interaction réciproque entre substrat technique et philosophie gestionnaire apparaît également très nettement lors de la relance de la mise en œuvre de la méthode au sein de la fonction RAE. Le SI ayant de nombreuses possibilités de paramétrage, de nombreuses discussions entre consultants et acteurs de Valeo concernent ces éléments. L'analyse de cette période nous permet de mettre en évidence l'émergence de nouveaux acteurs clés du processus d'appropriation. Cette analyse des acteurs permet alors de montrer comment l'appropriation pousse également à la transformation de l'organisation par l'innovation managériale. $\mathrm{Au}$ cours des échanges entre consultants et acteurs de Valeo, il est intéressant de noter l'importance du rôle émergent des rédacteurs de roadmaps dans la réflexion. En effet, nommés par les responsables d'axes, ils sont en charge de rédiger le contenu des roadmaps. Leur rôle est central dans le dispositif, d'autant plus que dans cette phase les roadmaps sont utilisées en partie pour l'audit des sites («Ceux qui sont légitimes, ce sont donc 
les rédacteurs qui peuvent rentrer dans le système», Directeur de l'audit interne de Valeo, 2010). Certaines roadmaps ont dû être réécrites suite à une remontée des opérationnels qui les considéraient parfois comme trop génériques, pas assez adaptées aux pratiques du terrain ou bien dont le contenu était difficilement compréhensible avec des exigences non atteignables. MNM Consulting a ainsi proposé de créer un comité de rédaction des roadmaps composé systématiquement des managers groupe, d'opérationnels pour ancrer la roadmap dans une perspective terrain mais aussi d'auditeurs pour éviter à la source des différences d'interprétation avec les sites lors des audits. Ce comité de rédaction a donc été sollicité à l'issue du pilote pour participer aux ajustements finaux de la plateforme avant le déploiement à l'ensemble des entités. Nous avons ainsi pu constater à partir de 2010 de nombreux échanges entre les consultants de MNM Consulting et le comité de rédaction afin de pouvoir inclure les modifications demandées par les opérationnels et les auditeurs de Valeo (création de rubriques, gestion des droits d'administrateurs, terminologie utilisée en anglais sur la plateforme, etc.). Ce comité a par exemple été mobilisé en 2010 pour arbitrer sur le fait qu'un livrable justifiant d'un item devait être rendu obligatoire avec un document à joindre sur la plateforme (fichier word, excel, pdf, etc.). Ce document sert alors de "preuve» pour valider un item. Cette décision prise lors du comité permet de concilier à la fois les exigences de reporting pour les responsables de sites et les auditeurs mais également de réduire les ambiguïtés sur les manières de valider l'item, ce dernier étant un élément de confirmation de la validation d'un niveau de la roadmap. Ainsi, certains rôles d'acteurs peuvent émerger, contribuant à redistribuer les pouvoirs au sein de l'organisation, et permettant d'ancrer l'innovation dans les pratiques de l'organisation, en légitimant les acteurs clés du dispositif. Ceux-ci ont un rôle particulier lié à leur maîtrise du contenu de la roadmap, qui est le cœur fondamental de la méthode. Cela leur permet également une interprétation plus fine de la philosophie gestionnaire de la méthode, tout en ayant une maîtrise importante du substrat technique. A ce titre, ils se révèlent être les meilleurs traducteurs entre philosophie et substrat technique, dans l'espace de discussion qui leur a été accordé par les différents acteurs. En effet le contenu des roadmaps se veut évolutif, dans la logique de la philosophie de l'amélioration continue, et les rédacteurs se trouvent alors être le contact privilégié des opérationnels qui remontent commentaires et suggestions sur le contenu de la roadmap. Ainsi, la dimension collaborative de partage de bonnes pratiques entre entités peut réellement prendre corps, au-delà des simples intentions et volontés des concepteurs de l'innovation managériale.

Il est également intéressant de constater que ces comités de rédaction incluent des auditeurs, qui sont donc directement impliqués dans la mise en œuvre de la méthode en lien avec les opérationnels. Cette interaction directe entre ces catégories d'acteurs dans le cadre de l'innovation managériale, participe à faire évoluer la perception de l'audit au sein de Valeo. En effet, l'enjeu de la méthode était de rompre avec la culture top-down de l'audit pour proposer un outil de progrès qui s'appuie sur une logique de dialogue entre acteurs (entre les entités, avec la hiérarchie), dans une perspective d'amélioration continue. Les auditeurs ainsi consultés dans la rédaction du contenu, mais aussi en contact avec des opérationnels pour mieux appréhender la réalité du terrain, comprennent davantage l'évolution du rôle d'auditeur prôné par l'innovation managériale. Cela contribue donc à faire de la métrique fournie par les roadmaps une base de discussion entre auditeurs et audités, qui perçoivent alors l'audit comme une occasion de progression robuste et non d'un simple contrôle parfois détaché de la réalité du terrain.

\section{Discussion}

L'analyse longitudinale de la construction de la méthode 5 steps au sein de Valeo centrée sur le développement du SI qui soutient sa mise en œuvre nous permet de mieux appréhender le processus d'innovation managériale dans sa dimension d'appropriation par les utilisateurs. Plusieurs contributions peuvent être retenues. Notre recherche montre que le substrat technique participe pleinement au processus d'appropriation lorsqu'il permet la traduction la plus fidèle possible de la philosophie portée par l'innovation managériale. L'enjeu majeur réside donc dans une imbrication isomorphique entre substrat technique et philosophie gestionnaire. L'appropriation de l'innovation managériale repose sur une construction progressive entre substrat technique et philosophie gestionnaire, influencée par les acteurs en présence dans cette négociation. Réciproquement, la construction de l'innovation peut transformer le rôle et le pouvoir des acteurs impliqués dans ce processus, et ainsi contribuer à l'évolution de l'organisation par l'émergence de ces nouveaux acteurs clés qui s'apparentent à des traducteurs et des relais de la philosophie gestionnaire. Cette interaction permet d'assurer le processus interprétatif qui favorise l'appropriation. En effet, l'appropriation passe par une interprétation et une construction de sens négociées entre les acteurs (Ségrestin, 2004). Notre étude de cas nous permet de dépasser les travaux sur les composants des innovations managériales (David, 1996; Mamman, 2009) dans ce processus de construction en proposant une réflexion sur l'interaction et l'enrichissement mutuel de deux composants particuliers. Ainsi, penser et construire le substrat technique concrètement et dans l'objectif d'appropriation conduit à redéfinir la philosophie de l'innovation. La confrontation à la pratique pousse à réfléchir à l'applicabilité de cette philosophie et conduit donc à la redéfinir, la préciser et l'expliciter. Réciproquement les défauts d'appropriation, de mauvaise compréhension de la philosophie conduisent à améliorer le substrat technique pour qu'il soit réellement porteur de la philosophie. La construction et l'amélioration du substrat technique visent alors à améliorer la traduction de la philosophie gestionnaire au sein du substrat technique. Ce dernier permet en quelque sorte une confrontation empirique de l'innovation managériale en l'expérimentant dans un processus d'essai-erreur. Ce résultat permet également de dépasser les travaux de Birkinshaw et al. (2008) en précisant davantage les interactions à l'œuvre entre les phases d'invention, de mise en œuvre et de théorisation du processus d'innovation managériale. En effet, ces interactions s'inscrivent dans une volonté d'alignement des concepteurs entre leur intention et la perception de l'innovation par les utilisateurs; cet alignement est assuré par un effort de réflexivité et d'apprentissage progressif de la part des concepteurs quant à la réalité de la mise en œuvre de l'innovation. 
Notre cas nous permet également de discuter les rôles des acteurs impliqués dans l'appropriation de l'innovation managériale, dans la lignée des travaux de Birkinshaw et al. (2008). En effet, l'interaction entre substrat technique et philosophie gestionnaire dans la construction de l'identité de l'innovation s'insère dans un contexte relationnel particulier dépendant des acteurs impliqués initialement dans l'innovation, mais aussi d'acteurs émergents au cours de la mise en œuvre de l'innovation. Plusieurs acteurs concourent à l'appropriation du fait d'une légitimité existante, notamment la direction de l'organisation (Damanpour et al., 2006; Birkinshaw et al. 2008; Peeters et al., 2014) mais l'innovation managériale peut aussi reconfigurer certaines relations entre acteurs plus opérationnels. Dans notre cas, le rôle de la DSI s'est nettement trouvé renforcé puisque l'innovation managériale est supportée dès sa conception par un SI. Le DSI étant l'un des concepteurs de la méthode, il a d'abord expérimenté la création des roadmaps de management au niveau des SI de Valeo et de ses équipes. Il a ensuite promu la méthode avec l'outil approprié auprès de la Direction Générale. Sa position hiérarchique au sein de Valeo et sa légitimité par rapport au substrat technique expliquent en partie le déploiement de 5 steps à l'ensemble du groupe Valeo. Cette légitimité peut également être mise en parallèle de la culture de l'organisation qui favorise le déploiement des méthodes portées par le groupe. On retrouve donc le rôle de la culture dans l'appropriation de l'innovation managériale (Volderba et al., 2010). L'appropriation est également favorisée par la légitimité émergente de nouveaux acteurs promus par les nouvelles relations favorisées par l'innovation, dans notre cas les rédacteurs des roadmaps. Ainsi, on constate que l'innovation est bien portée par les nouveaux acteurs définis dans cette nouvelle configuration, et non uniquement par les porteurs du changement (Alter, 2000). Ainsi, le pouvoir des rédacteurs a été légitimé au sein de l'organisation par la création des comités de rédacteurs, sollicités de manière plus générale dans la mise en œuvre de la méthode, et qui participent à une création de sens de l'innovation en phase avec l'intention des concepteurs, dans la relation entre substrat technique et philosophie gestionnaire. Lappropriation est donc facilitée par l'émergence d'un espace de discussion et d'échanges de bonnes pratiques autour de l'innovation managériale entre divers acteurs de l'organisation : DSI, rédacteurs, fonctionnels et direction. A l'image des communautés de pratiques, on retrouve ici une volonté des acteurs d'améliorer leur pratique et leur savoir-faire (Lave et Wenger, 1991). La conception progressive de l'innovation, et donc la création de connaissances autour de l'innovation, est en effet possible parce que ce type de communauté est le maillon fondamental permettant de passer d'un apprentissage individuel à un apprentissage collectif (Cohendet et al., 2003). C'est à ce niveau que les acteurs peuvent développer les représentations partagées de l'innovation. L'émergence d'une communauté traduit également l'émergence d'une identité en tant que groupe au sein de l'organisation. Cette identité se construit autour des pratiques et valeurs liées à l'innovation, qui sont discutées, négociées par les membres de la communauté et participe à la création de sens; et favorise l'appropriation de l'innovation managériale.

Notre cas souligne également le rôle des acteurs externes à Valeo dans l'appropriation. Il est particulièrement intéressant de discuter le rôle joué par les chercheurs dans ce processus, notamment dans la deuxième phase de conception du SI dédié à la mise œuvre de l'innovation. Ce SI visait clairement à améliorer l'appropriation de la méthode, dans la mesure où l'analyse menée en 2008 par deux chercheurs avait mis en évidence les défauts d'appropriation liés en grande partie à une mauvaise traduction de la philosophie par le SI mobilisé dans la première mise en œuvre de la méthode. La présence des chercheurs lors des réunions de travail dans cette conception du SI a eu plusieurs effets. Tout d'abord, leur présence a poussé à une meilleure explicitation des intentions des deux concepteurs. Afin de pouvoir travailler sur le SI, il était nécessaire de bien comprendre les objectifs visés en termes de management par ces concepteurs. A partir de cette meilleure compréhension des objectifs, les chercheurs ont pu orienter la conception du SI permettant une meilleure liaison entre substrat technique et philosophie gestionnaire. Ainsi, au-delà des apports de légitimité et d'expertise de ces acteurs externes (Birkinshaw et al., 2008), notre cas révèle qu'ils participent également à une meilleure traduction de la philosophie de l'innovation dans les dispositifs matériels qui portent l'innovation et qui seront donc les éléments directement utilisés par les acteurs de l'organisation.

Il est également possible de suggérer quelques implications managériales. La comparaison des deux situations d'appropriation de l'innovation managériale dans les périodes 1 et 3 nous permet de discuter des leviers qui favorisent cette appropriation et de la manière de l'évaluer. Tout d'abord, il est fondamental de faire émerger, et donc de penser en amont, un espace d'interaction entre substrat technique et philosophie gestionnaire, qui doivent co-évoluer progressivement et participer à la création d'un sens partagé de l'innovation managériale. Cet espace doit être déterminé d'un point de vue concret en termes d'espaces et de chronologie dans le déroulé du processus d'appropriation. Pour permettre cette interaction, les acteurs impliqués dans le processus doivent également être capables de comprendre et traduire une philosophie au-delà d'une dimension simplement matérielle de l'innovation; mais également d'interroger les philosophies sous-jacentes possibles dans l'utilisation du substrat technique et ses possibles détournements. Ensuite, l'émergence de nouveaux acteurs, dont le rôle est compris par l'organisation et en phase avec l'innovation managériale, apparaît donc comme un marqueur de l'appropriation. Mais il faut dépasser la simple désignation d'acteurs porteurs du changement liée à l'innovation et anticiper d'un point de vue organisationnel leur identification et leur légitimation en interne. Ces acteurs doivent se positionner à l'interstice de différentes fonctions et être pleinement intégrés dans des schémas organisationnels même si ceux-ci sont temporaires le temps de l'appropriation de l'innovation managériale. Ils doivent s'apparenter à des facilitateurs entre les co-concepteurs de l'innovation et contribuer à l'explicitation de la philosophie gestionnaire, tant en termes de traduction sémantique que de formalisation opérationnelle. Certaines organisations ont par exemple créé des entités comme les PMO (Project Management Office) qui ont pour objet de piloter la mise en place de projets transversaux et de référentiels en parallèle de la DSI. Ils fédèrent ainsi les différents co-concepteurs. L'appropriation se produit lorsqu'une communauté en capacité d'échanger sur les représentations et pratiques de l'innovation émerge. Elle est le garant d'un apprentissage collectif et du passage d'une utilisation individuelle à l'intégration 
dans les routines organisationnelles. L'organisation est alors en capacité de produire les nouvelles connaissances, qui réduisent l'écart entre l'innovation et l'organisation. L'appropriation d'une innovation doit donc s'inscrire dans un modèle politique (David, 1998) qui favorise la transformation des relations pour assurer l'implémentation de l'innovation. Les connaissances ne doivent pas seulement être produites par les concepteurs de l'innovation mais bien par les acteurs inscrits dans un nouveau jeu de relation.

\section{Conclusion, limites et voies de recherche}

Cette recherche permet de reconsidérer la dimension matérielle dans le processus d'innovation managériale, dans lequel la philosophie gestionnaire est vue comme le cœur de l'innovation. Elle met en évidence le rôle fondamental du substrat technique dans la construction du sens et le succès de l'innovation au sein de l'organisation qui la crée. Cette recherche comporte des limites inhérentes à la méthodologie choisie de l'étude de cas unique. Il faut considérer ces résultats au regard du choix du design méthodologique. La généralisation analytique est à replacer dans le genre de contexte du cas étudié. Notre cas repose sur un substrat technique particulier, un système d'information dans un contexte d'innovation managériale avec une forte proximité entre le DSI de Valeo et les équipes de MNM. L'innovation retenue accorde une place majeure au substrat technique avec un effort important de conception de celui-ci. Nos résultats peuvent aussi être contrastés par le fait que les roadmaps de management ont également d'abord été testées sur des problématiques internes à la DSI, ce qui a sans doute pu leur conférer une légitimité plus rapidement et facilement que si elles avaient été testées auprès d'une autre direction. Il conviendrait de réinterroger nos résultats dans le cas d'innovations managériales où la composante philosophie gestionnaire serait dominante et le substrat technique moins affirmé. Dans notre cas, le SI peut être aussi perçu comme une innovation technologique. Certains auteurs suggèrent ainsi d'explorer les liens entre l'innovation managériale et l'innovation technologique et leur contribution à la performance de l'organisation (Damanpour et al., 1989; Le Roy et al., 2013), notamment parce qu'un grand nombre d'innovations managériales sont centrées autour de nouvelles technologies d'information et de communication (Hecker et Ganter, 2013). De plus, nous ne pouvons pas totalement isoler l'effet substrat technique dans la compréhension de l'appropriation de l'innovation managériale. En effet, dans le cadre de la seconde mise en ouvre de la méthode au sein de Valeo, il existe nécessairement un effet apprentissage des acteurs de l'organisation qui concourt également à une meilleure compréhension des intentions des concepteurs. L'évolution de la méthode traduit aussi une nouvelle intention du management qui peut influencer ce processus.

Dans le prolongement de notre étude de cas, une analyse des innovations managériales pourrait être menée pour d'une part voir si elles reposent nécessairement pour la plupart sur un substrat technique dont les formes peuvent varier, et d'autre part, déterminer si nos résultats sont valables pour d'autres types de substrat technique qu'un système d'information. En effet, les propriétés du SI peuvent faciliter les évolutions et commodifications de l'innovation managériale. Une autre piste pourrait être la réalisation d'une échelle multidimensionnelle de mesure de l'appropriation d'une innovation managériale en prenant en compte les trois dimensions utilisées (substrat technique, philosophie gestionnaire et vision simplifiée des relations organisationnelles). Un prolongement de cette recherche pourrait être enfin une réflexion approfondie sur le processus de conception simultanée d'une innovation managériale et d'un SI, les deux étant souvent dissociés comme dans notre étude de cas alors que le SI peut être perçu lui-même comme une forme d'innovation.

\section{Bibliographie}

Abrahamson, E. (1996), «Management fashion», The Academy of Management Review, 21 (1), p. 254-285.

Alter, N. (2000), L'innovation ordinaire, Presses Universitaires de France, 2013, 6ème edition.

Ansari, S., Reinecke, J., SpaAn A. (2014), «How are Practices Made to Vary? Managing Practice Adaptation in a Multinational Corporation », Organization Studies, Vol. 35(9) p. 1313-1341.

Birkinshaw, J., Hamel, G., Mol, M.J. (2008), «Management innovation ", The Academy of Management Review, 33 (4), p. $825-845$.

Cohendet, P., Creplet, F., Dupouët, O. (2003), «Innovation organisationnelle, communautés de pratique et communautés épistémiques : le cas de Linux", Revue française de gestion, 2003/5, n¹46, p. $99-121$.

Damanpour, F., Aravind, D. (2012), «Managerial innovation : Conceptions, Processes and Antecedents", Management and Organization Review, $8: 2$, p. 423-454.

Damanpour, F., Schneider, M. (2006), «Phases of the Adoption of Innovation in Organizations : Effects of Environment, Organization and Top Managers ", British Journal of Management, vol. 17, p. 215-236.

Damanpour, F., Evan, W. M. (1984), «Organizational innovation and performance : the problem of 'organizational lag' ", Administrative Science Quarterly, 29 (3), p. 392-409.

DAvid, A. (1996), "Structure et dynamique des innovations managériales », Cahier du CGS n²12, juillet.

DAvid, A. (1998), «Outils de gestion et dynamique du changement», Revue Française de Gestion, sept-oct., p. 44-59.

Desanctis, G., Poole, M.S. (1994), «Capturing the complexity in advanced technology use : adaptative structuration theory", Organization Science, 5(2), p. 121-147.

Dubouloz, S., Bocquet, R. (2013), «Innovation organisationnelle. S'ouvrir pour innover plus ", Revue française de gestion, 2013/6, n²35, p. 129-147.

Dumez, H. (2013), Méthodologie de la recherche qualitative, Vuibert, $227 \mathrm{p}$.

Edquist, C., Hommen, C. L., Mckelvey, M. (2001), Innovation and employment : Process versus product innovation, Cheltenham : Edward Elgar.

EISENDHARDT, K. (1989), «Building theories from case study research ", Academy of Management Review, vol 14, p. $532-550$. 
FALL, I., (2008), Approche gestionnaire de la capacité organisationnelle et pilotage du progrès: Apports d'un dispositifpionnier de gestion des capacités organisationnelles dans une entreprise mondialisée, Thèse de doctorat, Ecole des Mines de Paris.

George, A. L., Bennett, A. (2005), Case Studies and Theory Development in the Social Sciences, MIT Press.

Gosselin, M. (1997), «The effect of strategy and organizational structure on the adoption of Activity-Based Costing", Accounting, Organizations and Society, 22(2), p. 105-122.

Grimand, A. (2012), «L'appropriation des outils de gestion et ses effets sur les dynamiques organisationnelles : le cas du déploiement d'un référentiel des emplois et des compétences", Revue Management \& Avenir, 2012/4, n 54, p. 237-257.

Hamel, G. (2006), «The why, what and How of Management Innovation ", Harvard Business Review, 84 : 2, p. 72-84.

Hamel, G. (2009), "Management innovation », Leadership Excellence, vol. 26, n5, p. 5 Hansen, M. T., Nohria, N., Tierney, T. (1999), "What's your strategy for managing knowledge? ", Harvard Business Review, March-April, p. 106-116.

Hatchuel, A. (2000), «Quel horizon pour les sciences de gestion? Vers une théorie de l'action collective» in Les nouvelles fondations des sciences de gestion, Eds David, A., Hatchuel, A., Laufer, R, Vuibert, p. 7-43

Hatchuel, A., Weil, B. (1992), L'expert et le système : gestion des savoirs et métamorphose des acteurs dans l'entreprise industrielle suivi de quatre histoires de systèmes-experts, Economica.

Hecker, A., Ganter, A. (2013), «The influence of product market competition on technological and management innovation : Firm-level evidence from a large scale survey", European Management Review, vol 10, p. 17-33.

JAY, J. (2013), «Navigating paradox as a mechanism of change and innovation in hybrid organizations", Academy of Management Journal, vol. 56, nº 1, p. 137-159.

Kimberly, J.R. (1981), «Managerial innovation», In Nystrom, P. \& Starbuck, W., Handbook of organizational design, p. 84-104, New York : Oxford University Press.

Langley, A., Smallman, C., Tsoukas, H., Van De Ven, A. H. (2013), « Process studies of change in organization and management : unveiling temporality, activity and flow", Academy of Management Journal, vol. 56, ${ }^{\circ} 1$, p. 1-13.

Lave J., Wenger E.C. (1991), Situated Learning : Legitimate Peripheral Participation, Cambridge University Press, New York, NY.

Le Roy, F., Robert, M., Giuliani, P. (2013), «L'innovation managériale. Généalogie, défis et perspectives », Revue Française de Gestion, $\mathrm{n}^{\circ}$ 235, p. 77-90.

Leonardi, P. M., Barley, S. R. (2010), "What's Under Construction Here? Social Action, Materiality, and Power in Constructivist Studies of Technology and Organizing», Academy of Management Annals, vol. 4, p. 1-51.

LEONARDI, P. M. (2013), «Theoretical foundations for the study of sociomateriality", Information and Organization, 23, p. 59-76.

Lillrank, P. (1995), "The transfer of management innovation from Japan», Organization Studies, 16(6), p. 971989.
LORINO, P. (2002), «Vers une théorie pragmatique et sémiotique des outils appliquée aux instruments de gestion», Working paper, ESSEC.

Moisdon, J-C. (1997), Du mode d'existence des outils de gestion, Editions Seli Arslan.

Mallet, C. (2006), «Innovation et mesure de l'appropriation des outils de gestion : proposition d'une démarche de construction d'un tableau de bord», Acte de colloque "En route vers Lisbonne», 9 et 10 novembre.

Mamman, B. (2009), «From management innovation to management practice», The international journal of organizational innovation, 2 (2), p. 22-60.

Mangolte, P.A. (1998), Le concept de routine organisationnelle: entre cognition et insitution, Thèse de doctorat, Université Paris Nord

Mol, M. J., Birkinshaw, J. (2009), «The sources of management innovation : when firms introduce new management practices", Journal of Business Research, 62(12), p. 1269-1280.

OrLIKowsKi, W. J. (1992), «The duality of technology: Rethinking the concept of technology in organizations», Organization Science, vol. $3, \mathrm{n}^{\circ} 3$, p. 398-427.

Orlikowski, W. J., ScotT, S. V. (2008), «Sociomateriality : Challenging the separation of technology, work and organization », The Academy of Management Annals, vol. 2, ${ }^{\circ}{ }^{1}$, p. 433-474.

ORLIKOWSKI, W. J. (2010), «The sociomateriality of organisational life : Considering technology in management research ", Cambridge Journal of Economics, 34, p. 125-141.

Peeters, C., Massini, S., Lewin, A. Y. (2014), «Sources of Variation in the Efficiency of Adopting Management Innovation : The Role of Absorptive Capacity Routines, Managerial Attention and Organizational Legitimacy", Organization Studies, Vol. 35(9), p. 1343-1371.

Poole, M. S., Desanctis, G. (1990), «Understanding the use of group decision support systems : The theory of adaptive structuration ", In J. Fulk \& C. Steinfeld (Eds). Organizations and Communication Technology. Newbury Park, Ca : Sage.

Proulx, S. (2001), «Les formes d'appropriation d'une culture numérique comme un enjeu d'une société du savoir», Actes du colloque COREVI, Gouvernance et usages d'internet : vers une nouvel environnement normative, Montréal.

RAuffet, P. (2010), Prise en compte des facteurs formels et contextuels dans la gestion des capacités organisationnelles. Application aux organisations matricielles, Thèse de doctorat, Ecole Centrale de Nantes.

RAVICHANDRAN, T. (2000), «Swiftness and intensity of administrative innovation adoption : An empirical study of TQM in information systems", Decision Sciences, 31(3), p. 691-724.

Rouleau, L., Allard-Poesi, F., Warnier, V. (2007), «Le management stratégique en pratiques ", Revue Française de Gestion, $2007 / 5, n^{\circ} 174$, p. $15-24$.

SANIDAs, E. (2005), Organizational innovations and economic growth: Organosis and growth of firms, sectors, and countries, Cheltenham : Edward Elgar.

Segrestin, D. (2004), Les chantiers du manager, Armand Colin, Paris. 
Stensaker, I. G., LANGley, A. (2010), « Change management choices and trajectories in a multidivisional firm», British Journal of Management, vol. 21, p. 7-27.

VAN DE Ven, A. H. (1986), «Central problems in the management of innovation", Management science, 32, 5, p. 590-607.

Volberda, H. W., Foss, N. J., Lyles, M. A. (2010), « PerspectiveAbsorbing the concept of absorptive capacity: How to realize its potential in the organization field», Organization Science, 21, p. 931-951.

De Vaujany F.-X. (2006), « Pour une théorie de l'appropriation des outils de gestion : vers un dépassement de l'opposition conception-usage» Management \& Avenir, 9, 3, p.109.
De Vaujany, F.X., Grimand, A. (2005), « Réflexion sur la place de la perspective appropriative au sein des sciences de gestion ", In de Vaujany, F-X. (ed.), De la conception à l'usage : vers un management de l'appropriation des outils de gestion, EMS, p. 223-234.

Wolfe, R. A. (1994), "Organizational Innovation : Review, Critique and Suggested Research Directions », Journal of management studies, 31, 3, p. 405-431.

YIn, R.K. (1989), Case Study Research, Design and Methods, Newbury Park, CA, Sage.

ZBARACKI, M. (1998), «The rhetoric and reality of Total Quality Management ", Administrative Science Quarterly, 43, p. 602-636. 


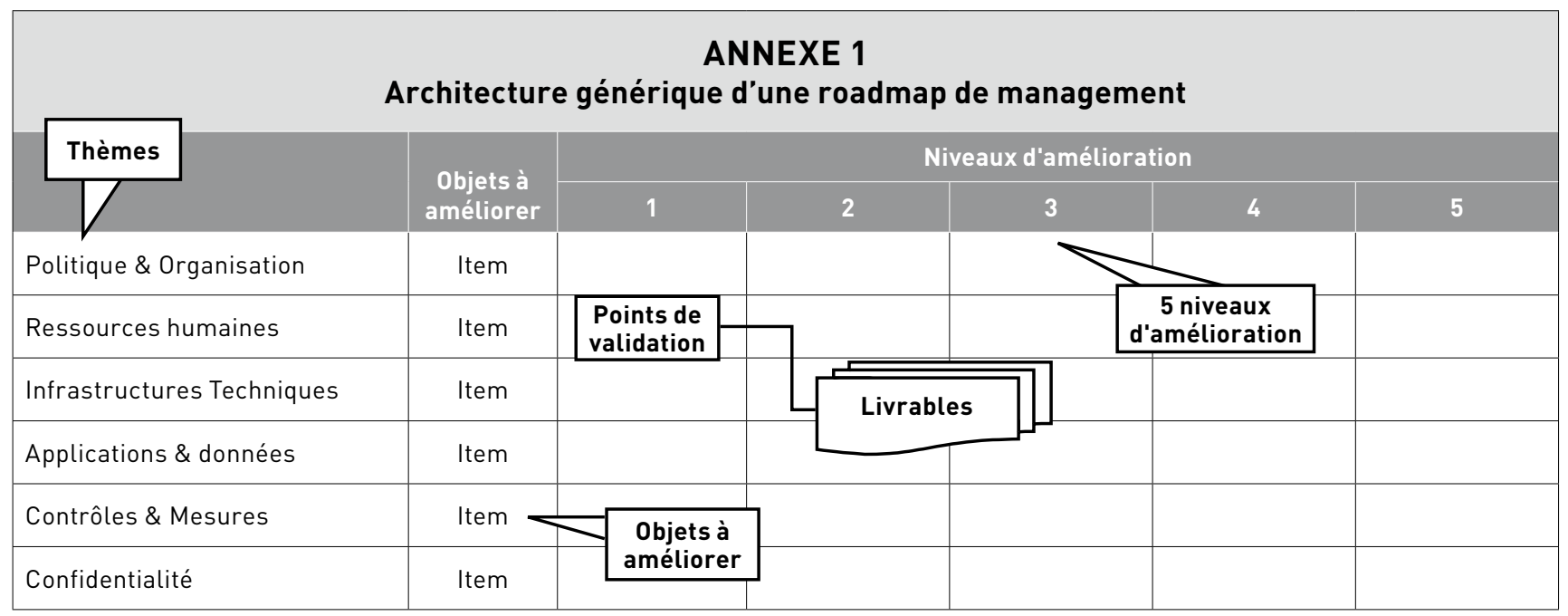




\section{ANNEXE 2 \\ Extraits de templates}

Premier type de template : analyse générale du processus d'innovation managériale (extraits)

\section{Exemple de codage phase 1}

Types de réunion/

participants/dates

Extraits sélectionnés des retranscriptions

Analyse/commentaires/ codage thématique

- La méthode 5 steps vise à se substituer au système d'audit classique des sites de Valeo. L'objectif est d'intégrer une logique de progressivité des entités à partir des roadmaps de management avec un selfassessment. L'intérêt pour Valeo est de simplifier et d'alléger la procédure d'audit et surtout de la rendre plus fiable, notamment dans l'évaluation du niveau de performance opérationnelle des entités.

- «Changement aussi dans l'utilisation : Précédemment, le questionnaire était un questionnaire d'audit, il était peu utilisé par les sites pour en faire un outil de progrès.»

- «On exige de la part des divisions qu'elles fassent du self assessment ».

- «La grosse différence pour moi aujourd'hui est que notre référentiel n'est plus un référentiel d'audit mais un outil de progrès pour les sites»

- «N'importe qui dans le groupe peut savoir le niveau de n'importe quel site par rapport à telle roadmap. Tout le monde peut tout savoir sur le voisin, pour se mesurer et pour comprendre pourquoi on n'y arrive pas »

- «Aujourd'hui pour chaque étape, vous avez la description de l'étape et des livrables c>est-à-dire que l'on demande un certain nombre de choses précises pour illustrer ce qui est décrit dans l'étape»

- «L'audit se fait tous les ans ou tous les 18 mois. C'est qu'on veut vérifier c'est si le self assessment est fiable ou pas.»

- «dans $80 \%$ des cas, il était assez simple de décrire les 5 niveaux et dans $20 \%$ des cas, il a fallu se creuser la tête pour arriver aux 5 niveaux»

- «Sinon grâce à la culture Valeo qui est forte, on est chez Valeo partout dans le monde; on ne remet pas souvent en question une méthodologie standard groupe.»

\section{Exemple de codage phase 2}

Réunion interne chez MNM

Consulting lle fondateur de MNM Consulting et le doctorant de l'IRCCYN $(28 / 05 / 2008)$
- Recherche sur l'agrégation des mesures concernant les roadmaps

- Développement d'une autre plate-forme full web

- Les opérationnels se tournent vers les auditeurs quand ils ont des questions

- Les roadmaps déploient une stratégie fonctionnelle

- Les roadmaps SI ont été les premières à être déployées

- Dans les entretiens beaucoup de réticences par rapport à Matrix loutil trop complexe)

- Outil déformé car à la base outil de PLM
Réunion interne chez MNM Consulting lle formateur de MNM Consulting pour 5 steps) (30/06/2008)
- Matrix est un produit PLM car disponible chez Valeo avec deux dimensions : dimension temps ( 5 assessment levels) et notion de cycle de vie (life cycle)

- Matrix est un «outil à la russe» : outil rustique qui marche mais il est assez complexe, soucis d'interface

- L'outil n'est pas adapté ergonomiquement et pas adapté en termes de fonctionnalités

- De très nombreux utilisateurs font des exports de roadmaps sous Excel

- Les utilisateurs ont beaucoup de livrables à gérer d'où l'utilisation d'Excel car plus simple

- Moteurs de l'utilisation : imposé par la hiérarchie, indexation financière des managers pour utiliser l'outil

- Projet de VRM 2 avec avantage de supprimer les licences et d'avoir une interface plus fonctionnelle
- Evolution de la culture chez Valeo qui est très marquée qui a permis de faire accepter l'innovation managériale. L'intérêt est d'être dans une philosophie gestionnaire moins top-down et plus d'échanges entre les entités avec une transparence sur les niveaux atteints.

- L'un des points importants de l'entretien est également la discussion des niveaux de détails des roadmaps et leur applicabilité à certains contextes opérationnels : questionnement sur le degré de granularité des éléments à fournir et des descriptions.

- Discussions sur les fonctions à développer autour de l'innovation managériale à partie du SI.

- Constat que l'outil utilisé Matrix pose des problèmes d'appropriation en raison de sa complexité et qu'il n'est pas conçu au départ pour la philosophie de l'innovation managériale.

- Le formateur et consultant de MNM Consulting ont abouti au constat que le premier SI Matrix n'est pas adapté à 5 steps et que la conception a été réalisée rapidement.

- Les problèmes ergonomiques entraînent l'utilisation d'excel pour les roadmaps de management.

- Le SI dédié (Roadmap Manager) a été travaillé sur de nouvelles fonctionnalités plus ergonomiques. 


\section{ANNEXE 2 \\ Extraits de templates}

Premier type de template : analyse générale du processus d'innovation managériale (extraits)

Exemple de codage phase 3

\begin{tabular}{|c|c|c|}
\hline $\begin{array}{l}\text { Réunion de } \\
\text { kick off avec } \\
3 \text { sponsors du } \\
\text { projet chez Valeo, } \\
\text { le directeur et une } \\
\text { consultante de } \\
\text { MNM consulting, } \\
\text { une consultante } \\
\text { d'une SSII } \\
(04 / 02 / 2010)\end{array}$ & $\begin{array}{l}\text { - Finalisation des } 2 \text { roadmaps «Santé \& Sécurité » et «Environnement» et } \\
\text { intégration dans l'outil RoadMap Manager. } \\
\text { - Intégration dans RoadMaps Manager de la partie gestion des audits. } \\
\text { - Proposition d'une démarche de déploiement pilote. } \\
\text { - Validation des dernières modifications (le cas échéant) sur les roadmaps } \\
\text { avec YH et intégration dans l'outil Roadmaps Manager. } \\
\text { - En fonction du pilote, réalisation de dernières modifications (le cas échéant) } \\
\text { sur les roadmaps et intégration dans l'outil Roadmaps Manager. } \\
\text { - Envoi des recommandations émises suite au déroulement du pilote à l'équipe } \\
\text { projet consultants. }\end{array}$ & $\begin{array}{l}\text { - Apparition d'un nouvel acteur } \\
\text { qui est une entreprise } \\
\text { européenne de services } \\
\text { informatiques qui va travailler } \\
\text { sur la mise en place de } \\
\text { Roadmap Manager. } \\
\text { - Définition des spécifications } \\
\text { pour l'expérimentation et } \\
\text { l'intégration des roadmaps } \\
\text { dans le nouvel SI. }\end{array}$ \\
\hline $\begin{array}{l}\text { Réunion de } \\
\text { kick off avec } \\
2 \text { sponsors du } \\
\text { projet chez Valeo, } \\
\text { le directeur et une } \\
\text { consultante de } \\
\text { MNM consulting, } \\
\text { une consultante } \\
\text { d'une SSII } \\
\text { (09/02/2010) }\end{array}$ & $\begin{array}{l}\text { - Cette réunion avait pour objectif d'aborder les problématiques de } \\
\text { Gouvernance en ce qui concerne les «Roadmaps et Audits EHS» dans le } \\
\text { cadre du projet de déploiement de Roadmap Manager } \\
\text { - Au regard de l'organisation des acteurs décrite dans le paragraphe } \\
\text { précédent, la gestion des droits sera organisée de la manière suivante au } \\
\text { sein de la solution Roadmap Manager (détails disponibles dans la note mais } \\
\text { confidentiel) }\end{array}$ & $\begin{array}{l}\text { - Expérimentation de roadmap } \\
\text { manager avec une réflexion } \\
\text { avancée sur la gestion des } \\
\text { droits utilisateurs grâce aux } \\
\text { fonctionnalités du nouveau SI : } \\
\text { accès en consultation et } \\
\text { modification à l'ensemble des } \\
\text { informations relatives aux } \\
\text { auto-évaluations, audits et } \\
\text { plans d'actions qui concernent } \\
\text { les branches } \\
\text { - La plateforme informatique doit } \\
\text { pouvoir aussi intégrer les plans } \\
\text { d'action. }\end{array}$ \\
\hline
\end{tabular}

\begin{tabular}{|c|c|c|c|c|c|}
\hline \multicolumn{6}{|c|}{$\begin{array}{l}\text { Deuxième type de template : Analyse de l'évolutions de la définition du SI à travers ses propriétés } \\
\text { et ses fonctionnalités et de la définition de l'identité de la méthode (extraits) }\end{array}$} \\
\hline $\begin{array}{l}\text { Matériau } \\
\text { d'analyse }\end{array}$ & $\begin{array}{l}\text { Type de } \\
\text { matériau }\end{array}$ & $\begin{array}{l}\text { Phase } \\
\text { concernée }\end{array}$ & Commentaires/analyse & $\begin{array}{l}\text { IM } \\
\text { comme } \\
\text { outil de } \\
\text { reporting }\end{array}$ & $\begin{array}{c}\text { IM } \\
\text { comme } \\
\text { outil de } \\
\text { pilotage et } \\
\text { de progrès }\end{array}$ \\
\hline $\begin{array}{l}\text { Discours du } \\
\text { dirigeant de MNM } \\
\text { Consulting en } \\
2007 \text { à Chartres } \\
\text { dans un séminaire } \\
\text { de travail } \\
\text { Présentation de la } \\
\text { méthode } 5 \text { steps }\end{array}$ & $\begin{array}{l}\text { Données } \\
\text { primaires }\end{array}$ & Phase 1 & $\begin{array}{l}\text { Le discours en } 2007 \text { de l'un des fondateurs de la méthode met } \\
\text { beaucoup plus l'accent sur la méthode } 5 \text { steps comme outil de } \\
\text { management qu'outil de reporting. Cela se traduit dans le nombre } \\
\text { d'occurrences de mots clés relatifs au management et également } \\
\text { à la couverture des mots clés dans l'ensemble de l'enregistrement. } \\
\text { La méthode permet de décrire les capacités organisationnelles et } \\
\text { de faire progresser l'organisation sur } 5 \text { niveaux (dans le discours } \\
\text { notions d'excellence, d'efficience, de performance, etc). De nombreux } \\
\text { passages sont également consacrés à l'opérationnalisation de la } \\
\text { méthode dans les organisations. }\end{array}$ & & $x$ \\
\hline $\begin{array}{l}\text { Guide utilisateur } \\
\text { de la première } \\
\text { version du SI pour } \\
\text { les roadmaps } \\
\text { (Matrix et SAP) }\end{array}$ & $\begin{array}{l}\text { Données } \\
\text { secondaires }\end{array}$ & Phase 1 & $\begin{array}{l}\text { Le guide utilisateur du premier SI (Matrix One et SAP) est en anglais } \\
\text { et comporte beaucoup plus de termes en rapport avec la méthode } \\
\text { comme outil de reporting que comme outil de management. Cela } \\
\text { ne traduit pas la philosophie gestionnaire d'un point de vue } \\
\text { sémantique. Le guide est par ailleurs très illustré de schémas } \\
\text { d'urbanisation simplifiés et de copies d'écran afin de décrire le SI } \\
\text { et son utilisation via son interface. Il ne figure à aucun endroit une } \\
\text { description ou un rappel de la philosophie gestionnaire orientée } \\
\text { vers } 5 \text { steps comme outil de management. }\end{array}$ & $x$ & \\
\hline $\begin{array}{l}\text { Entretien semi- } \\
\text { directif avec } \\
\text { le directeur de } \\
\text { l'audit interne de } \\
\text { Valeo en } 2006\end{array}$ & $\begin{array}{l}\text { Données } \\
\text { primaires }\end{array}$ & Phase 1 & $\begin{array}{l}\text { La méthode } 5 \text { steps vise à se substituer au système d'audit classique } \\
\text { des sites de Valeo. L'objectif est d'intégrer une logique de } \\
\text { progressivité des entités à partir des roadmaps de management } \\
\text { avec un self-assessment. L'intérêt pour Valeo est de simplifier et } \\
\text { d'alléger la procédure d'audit et surtout de la rendre plus fiable, } \\
\text { notamment dans l'évaluation du niveau de performance } \\
\text { opérationnelle des entités. }\end{array}$ & & $x$ \\
\hline
\end{tabular}




\section{ANNEXE 2 \\ Extraits de templates}

\begin{tabular}{|c|c|c|c|c|c|}
\hline \multicolumn{6}{|c|}{$\begin{array}{l}\text { Deuxième type de template : Analyse de l'évolutions de la définition du SI à travers ses propriétés } \\
\text { et ses fonctionnalités et de la définition de l'identité de la méthode (extraits) }\end{array}$} \\
\hline $\begin{array}{l}\text { Matériau } \\
\text { d'analyse }\end{array}$ & $\begin{array}{l}\text { Type de } \\
\text { matériau }\end{array}$ & $\begin{array}{l}\text { Phase } \\
\text { concernée }\end{array}$ & Commentaires/analyse & $\begin{array}{l}\text { IM } \\
\text { comme } \\
\text { outil de } \\
\text { reporting }\end{array}$ & $\begin{array}{c}\text { IM } \\
\text { comme } \\
\text { outil de } \\
\text { pilotage et } \\
\text { de progrès }\end{array}$ \\
\hline $\begin{array}{l}\text { Ouvrage la } \\
\text { méthode } 5 \text { steps } \\
\text { (117 pages) } \\
\text { Ouvrage rédigé les } \\
\text { deux fondateurs } \\
\text { de la méthode } \\
\text { (fondateur de } \\
\text { MNM Consulting } \\
\text { et le DSI de Valeo) } \\
\text { et paru en } 2008 \\
\text { aux éditions } \\
\text { AFNOR }\end{array}$ & \begin{tabular}{|l|} 
Données \\
secondaires
\end{tabular} & Phase 2 & $\begin{array}{l}\text { L'ouvrage } 5 \text { steps comporte plus de termes relevant de la méthode } \\
\text { comme un outil de management qu'un outil de reporting (notamment } \\
\text { en termes de degré de couverture pour le champs lexical). Le } \\
\text { chapitre } 1 \text { de l'ouvrage positionne d'emblée la méthode comme une } \\
\text { innovation managériale (c'est le titre). On retrouve la logique de } \\
\text { progression (le chapitre } 2 \text { est entièrement consacré à la notion de } \\
\text { progrès avec } 14 \text { pages) et le caractère opérationnalisable de la } \\
\text { méthode dans l'ensemble du livre. On est bien dans la présentation } \\
\text { de la philosophie gestionnaire des fondateurs avec plusieurs } \\
\text { illustrations. On constate qu'il n'est fait nulle part mention d'un } \\
\text { support SI pour déployer des roadmaps dans de très grandes } \\
\text { organisations. }\end{array}$ & & $x$ \\
\hline \begin{tabular}{|l} 
Etude de \\
panel avec \\
12 utilisateurs \\
de Valeo \\
(4 managers \\
et 8 ingénieurs \\
opérationnels)
\end{tabular} & $\begin{array}{l}\text { Données } \\
\text { primaires }\end{array}$ & $\begin{array}{l}\text { Phase } 2 \\
\text { (début) }\end{array}$ & $\begin{array}{l}\text { - L'outil répond globalement aux besoins du top management } \\
\text { (reportingl mais pas à ceux des opérationnels (pas assez un outil } \\
\text { de management, pas un outil pour faire des plans d'actions, ...) } \\
\text { - L'outil n'est pas intuitif, il ne permet pas d'effectuer les tâches } \\
\text { demandées facilement ni efficacement } \\
\text { - L'accès aux fenêtres principales loù l'utilisateur agit) n'est pas } \\
\text { aisé. La navigation est confuse, et le nombre de clics pour afficher } \\
\text { l'écran principal peut parfois atteindre la dizaine. }\end{array}$ & $x$ & \\
\hline $\begin{array}{l}\text { Plaquette } \\
\text { commerciale en } \\
\text { pdf de } 4 \text { pages de } \\
\text { MNM Consulting } \\
\text { sur Roadmap } \\
\text { Manager }\end{array}$ & $\begin{array}{l}\text { Données } \\
\text { secondaires }\end{array}$ & Phase 3 & $\begin{array}{l}\text { - La plaquette met clairement l'accent sur le SI comme outil de } \\
\text { management (sous-titre : «déployer des plans stratégiques»). } \\
\text { On retrouve également la logique de reporting avec deux rubriques } \\
\text { détaillées mais toujours en parallèle de l'idée d'un outil de } \\
\text { management (l'auto-évaluation et l'audit; les tableaux de bord). } \\
\text { - Roadmap Manager est également présenté comme une solution } \\
2.0 \text { p. } 3 \text { avec un accent mis au niveau de l'accessibilité laccès en } \\
\text { mode Saas) et de l'interopérabilité avec d'autres référentiels } \\
\text { (XML, CSV, LDAP). }\end{array}$ & $x$ & $x$ \\
\hline $\begin{array}{l}\text { Réunion avec } \\
3 \text { sponsors du } \\
\text { projet chez Valeo, } \\
\text { le dirigeant de } \\
\text { MNM consulting } \\
\text { et deux } \\
\text { consultants, } \\
\text { une consultante } \\
\text { d'une SSII } \\
\text { (février 2010) }\end{array}$ & $\begin{array}{l}\text { Données } \\
\text { primaires }\end{array}$ & Phase 3 & $\begin{array}{l}\text { - Réunion qui avait pour objectif d'aborder la structure et le contenu } \\
\text { des } 2 \text { roadmaps Santé, Sécurité et Environnement ainsi que de } \\
\text { sélectionner les sites qui seront dans le périmètre du pilote. } \\
\text { - On constate que la SSII est toujours présent aux réunions } \\
\text { permettant de paramétrer l'interface logicielle. } \\
\text { - Réflexion sur les types de contenus de la roadmap et les exigences } \\
\text { pour valider un niveau en s'appuyant sur les propriétés } \\
\text { informatiques afin de garantir la véracité des éléments. } \\
\text { - Cette évolution du SI intègre également une réflexion sur la } \\
\text { manière de consolider les données et de les présenter }\end{array}$ & & $x$ \\
\hline
\end{tabular}

